CONFORMAL GEOMETRY AND DYNAMICS

An Electronic Journal of the American Mathematical Society

Volume 7, Pages 49-75 (July 17, 2003)

S $1088-4173(03) 00084-5$

\title{
DISTINGUISHING PROPERTIES OF WEAK SLICE CONDITIONS
}

\author{
STEPHEN M. BUCKLEY AND ALEXANDER STANOYEVITCH
}

\begin{abstract}
The slice condition and the more general weak slice conditions are geometric conditions on Euclidean space domains which have evolved over the last several years as a tool in various areas of analysis. This paper explores some of the finer distinctive properties of the various weak slice conditions.
\end{abstract}

\section{INTRODUCTION}

The slice condition is a metric-geometric condition for domains in Euclidean spaces $\mathbb{R}^{n}$ that was introduced by the first author and Koskela [BK2] to obtain a set of geometric classifications of a large class of domains in Euclidean spaces which support any of the Sobolev imbeddings, for integrability index $p \geq n$. In later works $([\overline{\mathrm{BO}},[\mathrm{BS1}],[\mathrm{BS} 2])$, variations of the slice condition were used to refine these results and also to obtain unrelated results in other areas of analysis. There were many variants of these conditions such as (inner) $\alpha$-wslice and (inner) $\alpha$ wslice $^{+}$conditions, all of which were defined for a variety of purposes; see Section 2 for definitions. We refer to them generically as weak slice conditions, since $\alpha$-wslice conditions are strictly weaker than the slice condition. The index $\alpha$ indicates which metric is employed; the applications to Sobolev imbeddings require different metrics for each value of $p \geq n$.

All slice-type conditions, whether the slice condition or any weak slice condition, are very weak. For instance, they are all satisfied by every simply connected planar domain. This follows by the Riemann mapping theorem from the more general fact that the quasiconformal images of inner uniform domains in $\mathbb{R}^{n}$ satisfy the slice condition and all weak slice conditions (once a certain auxiliary parameter $C$ is sufficiently large); see Theorem 3.1 of [BS2]. Inner uniform domains, introduced by Väisälä $[\overline{V 5}$, generalize the well-known class of uniform domains.

In [BS2], the authors used weak slice conditions to obtain results regarding quasiconformal equivalence of product domains. One pleasing aspect of the main results of this work was that the weak slice conditions did not appear at all in the statement of the results, but rather as a tool in the proof. In [BO, Theorem 3.4], the slice condition is used to establish one-half of an equivalence in certain situations between a two-weighted variant of Trudinger's inequality and a certain global balance condition. Finally, in $[\mathrm{BB}]$, it is shown that under certain rather minimal assumptions, the metric spaces on which the associated quasihyperbolic metric is

Received by the editors October 9, 2001 and, in revised form, April 10, 2003.

2000 Mathematics Subject Classification. Primary 30C65, 46E35.

The first author was partially supported by Enterprise Ireland.

(C)2003 American Mathematical Society 
Gromov hyperbolic ( $k G$-hyperbolic spaces for short) coincide with the metric spaces that satisfy a variant of the slice condition, and that kG-hyperbolic spaces satisfy all types of (weak) slice conditions that were previously defined. This equivalence, as a by-product, proves a conjecture of Bonk, Heinonen, and Koskela [BHK] by showing that a Euclidean domain is a kG-hyperbolic space if and only if it satisfies two simpler conditions (separation and Gehring-Hayman).

With such a range of applications, it should be useful to have a solid understanding of (weak) slice conditions both in Euclidean and general metric space context. Many properties and examples of these conditions in the Euclidean context were obtained in [BS1] and [BS2, but some fundamental questions remain open even in this context. Several of these were presented in Section 6 of [BS2]. In this work we construct examples that fully answer one and partially answer another of these open problems, and we also answer another question that was not posed there, but is related to the results in [BS2]. In the process, we also develop some of the basic theory of wslice conditions in its most general metric space context.

After some basics in Section 1, we define and develop the basic theory of weak slice conditions in Section 2; we also partially solve one of the aforementioned open problems there. In Section 3, we present two types of examples that fully answer another of these open problems by showing that the class of domains satisfying a weak slice condition is strictly smaller if we use the inner Euclidean, rather than the Euclidean metric. The first example, a little simpler, will involve an imbedded 2 -manifold in $\mathbb{R}^{3}$. After this example, we shall give a planar domain example and sketch the additional details.

Inner uniform domains turn out to be a limiting class of domains called inner $\alpha$-mCigar domains, $0 \leq \alpha<1$ (case $\alpha=0$ ). These classes, whose definitions will be recalled in Section 4, increase strictly with $\alpha$. In Section 5 , we shall show that the hypotheses of the aforementioned Theorem 3.1 of [BS2] are about as weak as possible, in the sense that quasiconformal images of $\alpha$-mCigar conditions need not satisfy weak slice conditions when $\alpha>0$.

\section{Notation AND terminology}

Throughout this paper we will consistently employ the following notation:

$(\Omega, d)$ is an incomplete rectifiably connected metric space possibly subject to additional restrictions (it is often just a domain in Euclidean space), $\bar{\Omega}$ is its metric completion (viewed as a superset of $\Omega$ ), and $\partial \Omega=\bar{\Omega} \backslash \Omega$. For points $x, y \in \Omega$, a set $E \subset \Omega$, positive numbers $r, s$, we let:

$r \vee s$ denote the maximum of $r$ and $s$,

$r \wedge s$ denote the minimum of $r$ and $s$,

$\lceil r\rceil$ be the ceiling of $r$, i.e., the smallest integer $m \geq r$,

$\lfloor r\rfloor$ be the floor of $r$, i.e., the largest integer $m \leq r$,

$\operatorname{len}_{d}(E)$ denote the Hausdorff 1-dimensional measure of $E$ (so if $E$ is an arc, $\operatorname{len}_{d}(E)$ is just its arclength),

$\operatorname{dia}_{d}(E)$ denote the $d$-diameter of $E$,

$\delta_{\Omega}(x)$ denote the distance from $x$ to $\partial \Omega$,

$B_{d}(x, r)$ denote the open ball $\{y \in \Omega: d(x, y)<r\}$,

$B_{x}=B_{d}\left(x, \delta_{\Omega}(x)\right)$, and 
$\Gamma_{\Omega}(x, y)$ denote the class of all rectifiable paths $\lambda:[0, t] \rightarrow \Omega$ for which $\lambda(0)=x$ and $\lambda(t)=y$. Whenever $\lambda$ is a path, $\lambda^{*}$ denotes its image set. Whenever $E$ is an open or closed ball, $t E$ denotes its concentric dilate by a factor $t>0$.

For $\alpha \in[0,1]$ we will also make extensive use of the following subhyperbolic lengths and the corresponding metrics. For any rectifiable path $\gamma$ in $\Omega$, we define these quantities by

$$
\begin{aligned}
\operatorname{len}_{\alpha, \Omega}(\gamma) & =\int_{\gamma} \delta_{\Omega}^{\alpha-1}(z) d s(z), \\
d_{\alpha, \Omega}(x, y) & =\inf _{\gamma \in \Gamma_{\Omega}(x, y)} \operatorname{len}_{\alpha, \Omega}(\gamma),
\end{aligned}
$$

where $d s$ denotes the arclength measure. These concepts can then also be defined as one-dimensional Hausdorff measures on sets, so that $\operatorname{len}_{\alpha, \Omega}(\gamma)=\operatorname{len}_{\alpha, \Omega}\left(\gamma^{*}\right)$ if $\gamma$ is injective. We note that if $\Omega$ is a domain in Euclidean space, or in an imbedded $k$-manifold in $\mathbb{R}^{n}$, then $\operatorname{len}_{0, \Omega}$ and $d_{0, \Omega}$ are the well-known quasihyperbolic length and quasihyperbolic distance, and $d_{1, \Omega}$ is the inner metric with respect to $\Omega$. For brevity, we shall denote the inner metric on $\Omega$ as $d_{\Omega}$ and the corresponding inner diameter of a subset $E$ of $\Omega$ as $\operatorname{dia}_{\Omega}(E)$ in such cases. We shall also write $k_{\Omega}(x, y)$ in place of $d_{0, \Omega}(x, y)$.

Let us call $\gamma \in \Gamma_{\Omega}(x, y)\left(\alpha, C_{1}, C_{2}\right)$-efficient, or simply $\alpha$-efficient, if $\operatorname{len}_{\alpha, \Omega}(\gamma) \leq$ $\left(1+C_{1}\right) d_{\alpha, \Omega}(x, y)+C_{2}$. We say that $\gamma \in \Gamma_{\Omega}(x, y)$ is an $\left(\alpha, C_{1}, C_{2}\right)$-quasigeodesic for $x, y$ if $\gamma$ and all its subpaths are $\left(\alpha, C_{1}, C_{2}\right)$-efficient, while we say that $\gamma$ is an $\alpha$-geodesic if it is $(\alpha, 0,0)$-efficient (or equivalently an $(\alpha, 0,0)$-quasigeodesic). We say that $\gamma$ is a rough $\left(\alpha, C_{2}\right)$-geodesic if it is $\left(\alpha, 0, C_{2}\right)$-efficient (or equivalently an $\left(\alpha, 0, C_{2}\right)$-quasigeodesic). Obviously, efficient paths and rough geodesics exist, with $C_{1}, C_{2}$ as close to zero as we wish (we can even take one of the two parameters to be zero), but $\alpha$-geodesics might not exist. For instance in the Euclidean case, $\alpha$-geodesics exist if $\alpha=0$, but might not if $\alpha>0$; see GO and BS1. Example 1.2].

Let $C \geq 1, x, y \in \Omega$, and let $\gamma \in \Gamma_{\Omega}(x, y)$ be a path of length $l$ which is parametrized by arclength. We say that $\gamma$ is a $C$-uniform path for $x, y \in \Omega$ if it satisfies the bounded turning condition $l \leq C d(x, y)$ and the cigar condition $t \wedge(l-t) \leq C \delta_{\Omega}(\gamma(t))$. In this case, we get the estimates

$$
d_{\alpha, \Omega}(x, y) \leq \begin{cases}4 C^{2} \log \left(1+\frac{d(x, y)}{\delta_{\Omega}(x) \wedge \delta_{\Omega}(y)}\right), & \alpha=0, \\ C^{\prime}\left[\delta_{\Omega}(x) \vee \delta_{\Omega}(y) \vee d(x, y)\right]^{\alpha}, & \alpha>0,\end{cases}
$$

where $C^{\prime}=C^{\prime}(C, \alpha)$. The $\alpha>0$ case follows by an easy integration, estimating distance to the boundary by the triangle inequality for the initial and final parts of the path that are close to $x$ and $y$, respectively, and by uniformity for the rest of the path. The case $\alpha=0$ is Lemma 2.13 of [BHK].

We say that $\Omega$ is a $C$-uniform space if there is a $C$-uniform path for every pair $x, y \in \Omega$. We shall say more about such spaces in the Euclidean case in Section 4. For more on uniform domains in a general context, we refer the reader to [BHK].

\section{The WEAK SLICE CONDitions}

In this section we define and discuss weak slice conditions for an incomplete rectifiably connected metric space $(\Omega, d)$. In the Euclidean context, some of the material presented below is to be found in BS1] and BS2], although this section is 
essentially self-contained. One notable new result is Theorem 2.14 which partially answers an open question in [BS2].

For some results, we need $(\Omega, d)$ to satisfy two additional properties, local quasiconvexity and local external connectivity. Suppose $C \geq 1$. We say that $\Omega$ is locally $C$-quasiconvex if for every pair of points $x, y \in \Omega, y \in C^{-1} B_{x}$, there exists a path $\gamma \in \Gamma_{\Omega}(x, y)$ such that $\operatorname{len}_{d}(\gamma) \leq C d(x, y)$. We say that $\Omega$ is locally $C$-externally connected if, for every $z \in \Omega, 0<r<\delta_{\Omega}(z) / C$, and $x, y \in \Omega \backslash B_{d}(z, r)$, the path family $\Gamma_{\Omega \backslash B_{d}(z, r / C)}(x, y)$ is non-empty. We say $\Omega$ is locally $\left(C, C^{\prime}\right)$-nicely connected if it is both locally $C$-quasiconvex and locally $C^{\prime}$-externally connected. The local external connectivity property is a local (i.e. restricted radius) version of a condition that is nowadays usually referred to as LLC-2 in the literature, since it was originally the second part of the so-called linear local connectivity condition of Gehring and Martio [GM2, 2.9].

There are many locally nicely connected spaces. For instance, proper subdomains of $\mathbb{R}^{n}$ are locally $(1,1)$-nicely connected with respect to the Euclidean or inner Euclidean metric, or any metric intermediate between them (this is the class of metrics considered in [BS1] and [BS2]). More generally, it follows from Theorem 3.13 of [HK] that if $\Omega$ is an open connected set in a complete, Ahlfors upper regular, Loewner space $\left(X, d_{X}, \mu\right)$ of Hausdorff dimension $Q>1$, then $\Omega$ is locally nicely connected. Examples of Loewner spaces include Euclidean space, $n$-regular Riemannian manifolds of non-negative Ricci curvature, and Carnot-Carathéodory spaces such as the Heisenberg group; see [HK, Section 6]. The metric on $\Omega$ in these Loewner examples is the restriction of $d_{X}$ but note that the local $\left(C, C^{\prime}\right)$-nice connectivity condition for this metric implies a local $\left(C^{2}, C^{\prime}\right)$-nice connectivity condition with respect to the "inner" metric $d$ where $d(x, y), x, y \in \Omega$, is defined to be the infimum of $\operatorname{len}_{d_{X}}(\gamma)$, as $\gamma$ ranges over all paths in $\Gamma_{\Omega}(x, y)$ (thus generalizing the inner Euclidean case).

We are now ready to define our weak slice conditions, which depend on parameters $0 \leq \alpha<1 \leq C$. First, a collection $\mathcal{F}=\left\{S_{i}\right\}_{i=1}^{m}, m \geq 0$, of pairwise disjoint open subsets of $\Omega$ is a set of $(\alpha, C)$-wslices for $x, y \in \Omega$ and the associated collection of positive numbers $\left\{d_{i}\right\}_{i=1}^{m}$ is a set of $(\alpha, C)$-wnumbers if, for all $1 \leq i \leq m$, $d_{i} \geq \operatorname{dia}_{d}\left(S_{i}\right)$ and

$$
\begin{gathered}
\forall \lambda \in \Gamma_{\Omega}(x, y): \quad \operatorname{len}_{d}\left(\lambda^{*} \cap S_{i}\right) \geq d_{i} / C, \\
S_{i} \cap\left[C^{-1} B_{x} \cup C^{-1} B_{y}\right]=\emptyset .
\end{gathered}
$$

We refer to $\left\{\left(S_{i}, d_{i}\right)\right\}_{i=1}^{m}$ collectively as an $(\alpha, C)$-admissible set for the pair $x, y \in \Omega$. Note that $m=0$ is allowed in this definition: the empty set is trivially an $(\alpha, C)$ admissible set for every $x, y \in \Omega$. Next, we define $\mathrm{WS}_{\alpha}(x, y ; \Omega ; C)$ by

$$
\begin{aligned}
& \mathrm{WS}_{\alpha}(x, y ; \Omega ; C)=\sup \left\{\delta_{\Omega}^{\alpha}(x)+\delta_{\Omega}^{\alpha}(y)+\sum_{i=1}^{m} d_{i}^{\alpha}:\right. \\
&\left.\left\{d_{i}\right\}_{i=1}^{m} \text { is a set of }(\alpha, C) \text {-wnumbers for } x, y \in \Omega\right\} .
\end{aligned}
$$

Since the empty set is $(\alpha, C)$-admissible, it follows that $\mathrm{WS}_{\alpha}(x, y ; \Omega ; C)$ is at least equal to $\delta_{\Omega}^{\alpha}(x)+\delta_{\Omega}^{\alpha}(y)$. A priori, $\mathrm{WS}_{\alpha}(x, y ; \Omega ; C)$ could possibly be infinite, but, at least in the case of locally externally connected spaces, we shall prove that it satisfies a condition of the form

$$
\mathrm{WS}_{\alpha}(x, y ; \Omega ; C) \leq C^{\prime}\left[\delta_{\Omega}^{\alpha}(x)+\delta_{\Omega}^{\alpha}(y)+d_{\alpha, \Omega}(x, y)\right] .
$$


We define an $\alpha$-wslice space essentially by reversing this last inequality for large subhyperbolic distance. More precisely, we say that the pair $x, y$ satisfy an $(\alpha, C)$ wslice condition, $C \geq 1$, if

$$
d_{\alpha, \Omega}(x, y) \leq C \mathrm{WS}_{\alpha}(x, y ; \Omega ; C),
$$

and we say that $\Omega$ is a (two-sided) $(\alpha, C)$-wslice space if all pairs of points in $\Omega$ satisfy an $(\alpha, C)$-wslice condition]. Note that when $\alpha=0$, (WS-3) simply says that $\operatorname{len}_{0, \Omega}(\gamma) \leq C\left(2+m_{0}\right)$, where $m_{0}$ is the cardinality of the largest $(0, C)$ admissible set for the pair $x, y$. Note that in light of (WS-1), each of the slices $S_{i}$ must separate $x$ from $y$ in $\Omega$. If $\Omega \subsetneq \mathbb{R}^{n}$ is a domain, we call $\Omega$ an $(\alpha, C)$ wslice, or inner $(\alpha, C)$-wslice, domain if it is an $(\alpha, C)$-wslice space with respect to the Euclidean or inner Euclidean metric, respectively. Similarly, we talk of wslice and inner wslice manifolds when $\Omega$ is an imbedded $k$-manifold in $\mathbb{R}^{n}$. Notice that the difference between Euclidean and inner Euclidean $\alpha$-wslice domains is rather minor since distance to the boundary and the associated subhyperbolic metrics are unchanged, so that each of (WS-1) through (WS-3) has an identical form. The only change is in the requisite lower bound in the size of the $d_{i}$ 's (from $\operatorname{dia}\left(S_{i}\right)$ to $\operatorname{dia}_{\Omega}\left(S_{i}\right)$ ). Nevertheless, we shall see in the next section that there are indeed wslice domains that are not inner wslice domains.

It is also convenient to say that an $(\alpha, C)$-admissible set $\left\{\left(S_{i}, d_{i}\right)\right\}_{i=1}^{m}$ for $x, y \in \Omega$ is an $(\alpha, C)$-wslice dataset for $x, y$ if we additionally have the following condition:

$$
d_{\alpha, \Omega}(x, y) \leq C\left(\delta_{\Omega}^{\alpha}(x)+\delta_{\Omega}^{\alpha}(y)+\sum_{i=1}^{m} d_{i}^{\alpha}\right) .
$$

If the wnumbers are not specified, it is assumed that $d_{i}=\operatorname{dia}_{d}\left(S_{i}\right)$.

The definition of an (inner) wslice domain in $\mathbb{R}^{n}$ is unchanged (except for a controlled increase in $C$ ) if we insist that $d_{i}=\operatorname{dia}_{d}\left(S_{i}\right)$; this is obvious if $\alpha=0$, and follows from [BS1, Lemma 2.5] when $\alpha>0$. However, allowing inequality is often convenient. Oftentimes the value of the constant $C$ is unimportant and so we will, on such occasions, refer simply to " $\alpha$-wslice conditions and/or domains". Modulo a possible augmentation of $C$, condition (WS-2) can actually be dropped in the Euclidean setting if $\alpha>0$, but it is essential in case $\alpha=0$, lest every domain be a $(0, C)$-wslice domain; see [BS2, Theorem 5.1].

We next wish to examine some additional hypotheses which have turned out to be useful in [BS1] and [BS2]. It is assumed below that we already have an $(\alpha, C)$ wslice dataset $\left\{S_{i}, d_{i}\right\}_{i=1}^{m}$ for a pair of points $x, y \in \Omega$, and these conditions are assumed to hold for all $i, 1 \leq i \leq m$ :

$$
\begin{aligned}
\exists \gamma \in \Gamma_{\Omega}(x, y): & \operatorname{len}_{\alpha, \Omega}\left(\gamma^{*} \cap S_{i}\right) \leq C d_{i}^{\alpha}, \\
\exists z_{i} \in S_{i}: & B_{i} \equiv B_{d}\left(z_{i}, d_{i} / C\right) \subset S_{i}, \\
\forall \lambda \in \Gamma_{\Omega}(x, y): & \operatorname{dia}_{d}\left(\lambda^{i}\right) \geq d_{i} / C,
\end{aligned}
$$

where $\lambda^{i}$ denotes a component of $\lambda^{*} \cap S_{i}$ of maximal diameter. We will also consider the following condition which is one of the assumptions in the original slice condition in BK2, but is not a part of the 0 -wslice condition, and is mainly useful

${ }^{1}$ In [BS1] and [BS2], the labels (WS-2) and (WS-3) were reversed, but that does not suit our more general discussion here. 
in conjunction with (WS-4), with the same path $\gamma$ :

$$
\exists \gamma \in \Gamma_{\Omega}(x, y) \quad \forall z \in \sigma_{i} \equiv \gamma^{*} \cap S_{i}: d_{i} / C \leq \delta_{\Omega}(z) \leq C d_{i} .
$$

As in the earlier papers, we refer to $(\alpha, C)$-wslice spaces (or domains) which can be made to satisfy (WS-5) and (WS- $\left.1^{+}\right)$as $(\alpha, C)$-wslice ${ }^{+}$spaces (or domains).

In typical natural examples of slice domains, the slices satisfy these extra conditions also but in general, this need not be so. We shall say more about this below, but first let us pause to give some simple and preliminary estimates, examples, and counterexamples related to slice domains.

2.2. Estimates, examples, and counterexamples. In the general metric space setting, with $x, y \in \Omega$, we have the estimates

$$
\mathrm{WS}_{\alpha}(x, y ; \Omega ; C) \geq \begin{cases}2 \vee \log _{2}\left(\frac{d(x, y)}{\delta_{\Omega}(x) \wedge \delta_{\Omega}(y)}\right), & \alpha=0, \\ {\left[\delta_{\Omega}(x) \vee \delta_{\Omega}(y) \vee d(x, y)\right]^{\alpha},} & \alpha>0,\end{cases}
$$

whenever $C \geq 4$. Together with (1.1), it follows that uniform spaces are $\alpha$-wslice spaces for all $\alpha$.

By swapping $x$ and $y$ if necessary, it suffices to prove (2.3) under the assumption that $\delta_{\Omega}(x) \leq \delta_{\Omega}(y)$. In the case $\alpha=0$ (illustrated in Figure 2.4), we then pick as a set of wslices the concentric annuli with geometrically increasing radii $A_{i}=$ $B\left(x, 2^{i} \delta_{\Omega}(x)\right) \backslash B\left(x, 2^{i-1} \delta_{\Omega}(x)\right)$ for $1 \leq i \leq m_{0}$, where

$$
m_{0}=\left\lfloor\log _{2}\left[\frac{d(x, y)}{\delta_{\Omega}(x) \wedge \delta_{\Omega}(y)}\right]\right\rfloor-1 .
$$

In the case $\alpha>0$, the same set of slices works, but there is a much simpler dataset that also works: we pick zero slices if $d(x, y)<\delta_{\Omega}(x)+\delta_{\Omega}(y)$, or else we pick the single slice $B(x, d(x, y)) \backslash\left(\bar{B}\left(x, \delta_{\Omega}(x) / 2\right) \cup \bar{B}\left(y, \delta_{\Omega}(y) / 2\right)\right)$.

One typical type of slice $S$ for the points $x, y \in \Omega$, and any of the weak slice conditions, is an open subset of $\Omega$ whose "thickness" is comparable with its diameter, and such that the intersection of some quasihyperbolic geodesic $\gamma$ with $S$ is of bounded quasihyperbolic diameter. Let $\widetilde{\gamma}$ be the part of $\gamma$ that lies outside the balls $C^{-1} B_{x}$ and $C^{-1} B_{y}$ for some fixed $C>1$. If we can cover at least a fixed fraction (measured by quasihyperbolic length) of $\widetilde{\gamma}$ by such (disjoint) slices $S$, with uniform control over the constants involved, an $\alpha$-wslice condition will follow for all $\alpha$. When proving the $\alpha=0$ case of (2.3), we covered about half (or more) of the quasihyperbolic length of $\widetilde{\gamma}$ by slices of this type. The same type of slices allow one to see intuitively that all simply connected planar domains should be $\alpha$-wslice domains for all $\alpha$; see Figure 2.5. For a formal proof of a more general result, see BS2, Theorem 3.6]. To make Figure 2.5 unambiguous, we note that all slices are the connected components bounded by dotted curves; labels for the six small ones around the bottleneck are omitted.

So far we have given examples of domains that are $\alpha$-wslice domains for all $\alpha$. Rather complicated examples are given in [BS2, Section 4] to prove that the classes of $\alpha$-wslice domains are different for each $\alpha$. To help the reader get a better intuitive sense of wslice conditions, we give three much simpler examples here: one that is an $\alpha$-wslice domain only when $\alpha>0$, and two that are not $\alpha$-wslice domains for any $\alpha$. 


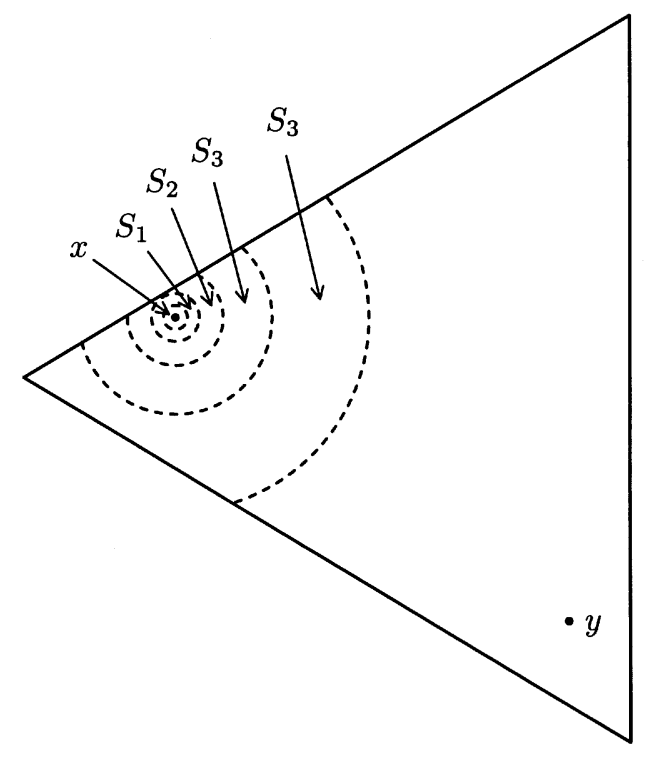

FIgURE 2.4. Concentric annular slices used to prove (2.3)

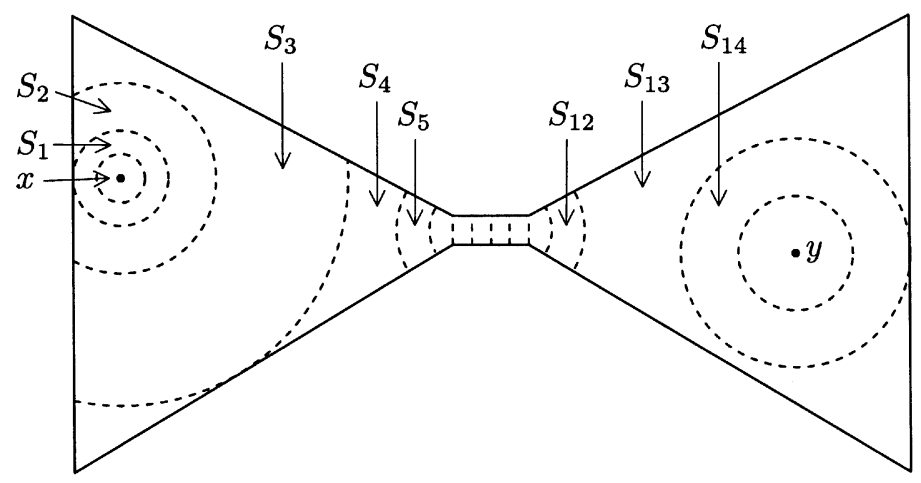

FiguRE 2.5. Slices in a simply connected planar domain

Consider the type of domain $\Omega$ illustrated in Figure 2.6, where a countable set $E$ is removed from a triangle $G$ in the $x y$-plane with vertex at the origin and baseline at $x=1$. Specifically, for each $k \in \mathbb{N}, n_{k}$ equally spaced points are removed from the interval given by the intersection of $G$ with the line $\pi(x)=2^{-k}$, where $\pi$ denotes projection onto the first coordinate in the plane. We assume that the sequence $\left(n_{k}\right)_{k=1}^{\infty}$ is unbounded. According to Proposition 4.5 of [BS1], every such domain is $\alpha$-wslice for all $\alpha>0$, but is not a 0 -wslice domain; we give a different justification of this fact after Corollary 2.9 below.

If we take out many more points, we can construct a domain that is not an $\alpha$-wslice domain for any $\alpha$. For instance, suppose we start off with the same planar triangle $G$ but now let $\Omega^{\prime}=G \backslash E^{\prime}$, where $E^{\prime}$ is a countable set chosen so that $\delta_{\Omega^{\prime}}(z) \leq 2^{-k !}$, whenever $z \in \Omega, \pi(z)<2^{-k}$. We also assume that the origin is the 


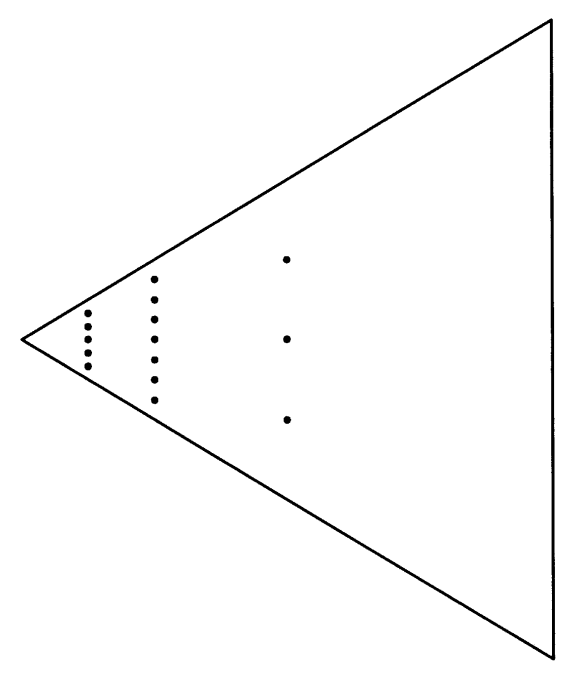

FiguRE 2.6. $\alpha$-wslice for $\alpha>0$, but not 0 -wslice

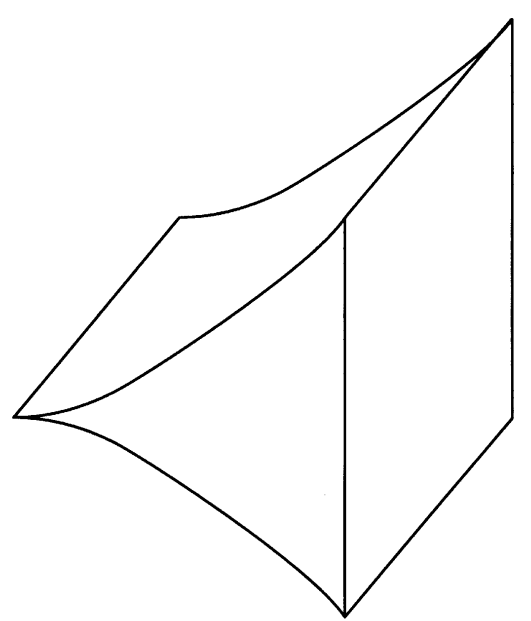

Figure 2.7. A cusped non- $\alpha$-wslice domain

only point of accumulation of $E^{\prime}$. We shall see soon that any such domain $\Omega^{\prime}$ fails to be an $\alpha$-wslice domain for each $\alpha$.

Another type of domain that does not satisfy any $\alpha$-wslice condition is any cusped domain in $\mathbb{R}^{n+1}, n \geq 2$, like the one in Figure 2.7. In fact, we can take any domain of the form $D \times(0,1)$, where $D$ is any Steiner symmetric cusp domain

$$
\left\{\left(x_{1}, x^{\prime}\right) \in \mathbb{R} \times \mathbb{R}^{n-1}: 0<x_{1}<1,\left|x^{\prime}\right|<\phi\left(x_{1}\right)\right\},
$$

$\phi$ being a strictly increasing continuous function with $\liminf _{t \rightarrow 0^{+}} \phi(t) / t=0$. According to the proof of BS1, Proposition 4.6], such domains $D$ are never " $\alpha$-mcigar domains" (a subclass of $\alpha$-wslice domains that we consider later in this paper), and it then follows from Theorem 4.1 of $\mathrm{BS2}$ that $D \times(0,1)$ can never be an $\alpha$-wslice domain. 
We now prove a simple but useful estimate which is easily seen to fail in our most general metric space setting, but is valid for locally externally connected spaces.

Lemma 2.8. Let $c \in(0,1]$ and $C \geq 2$ be constants. Suppose that $\Omega$ is a locally $C$-externally connected space, and that the set $S \subset \Omega \backslash\left(c B_{x} \cup c B_{y}\right)$ satisfies the separation condition

$$
\forall \lambda \in \Gamma_{\Omega}(x, y): \quad \lambda^{*} \cap S \neq \emptyset .
$$

Then $\delta_{\Omega}(z) \leq\left(C^{2} \vee(2 C / c)\right) \operatorname{dia}_{d}(S)$ for every $z \in S$.

Proof. Let $z \in S$. We claim that $d(x, z) \geq r \equiv\left[(c / 2) \wedge C^{-1}\right] \delta_{\Omega}(z)$. We may assume that $d(x, z) \leq C^{-1} \delta_{\Omega}(z)$. Since $C \geq 2$, this implies that $\delta_{\Omega}(z) \leq 2 \delta_{\Omega}(x)$. Since $d(x, z)>c \delta_{\Omega}(x)$, the claim now follows. By symmetry, we can replace $x$ with $y$ in the above argument to obtain $d(y, z) \geq r$.

Contrasting the local external connectivity condition (using the above choices of $x, y, z, r)$ with the separation property, we deduce that $S$ must have diameter larger than $r / C$.

Corollary 2.9. Let $c \in(0,1], \alpha \in[0,1)$, and $C \geq 2$ be constants, let $x, y$ be points in a locally $C$-externally connected space $\Omega$, and let $E \subset \Omega$. Suppose that $\left\{S_{i}\right\}_{i=1}^{m}$ is a set of bounded pairwise disjoint subsets of $\Omega \backslash\left(c B_{x} \cup c B_{y}\right)$ such that $\operatorname{len}_{d}\left(E \cap S_{i}\right) \geq c d_{i}$ for some numbers $d_{i} \geq \operatorname{dia}_{d}\left(S_{i}\right)$. Furthermore, we assume that each $S_{i}$ satisfies the separation condition: $\lambda^{*} \cap S_{i} \neq \emptyset$ for all $\lambda \in \Gamma_{\Omega}(x, y)$. Then there exists $C^{\prime}=C^{\prime}(c, \alpha, C)$ such that $d_{i}^{\alpha} \leq C^{\prime} \operatorname{len}_{\alpha, \Omega}\left(E \cap S_{i}\right)$ for each $1 \leq i \leq m$. Consequently, $\sum_{i=1}^{m} d_{i}^{\alpha} \leq C^{\prime} \operatorname{len}_{\alpha, \Omega}(E)$.

This corollary follows easily from the lemma. The last statement in the corollary immediately implies (2.1), by taking $E=\gamma^{*}$ for some $(\alpha, 1,0)$-efficient $\gamma \in \Gamma_{\Omega}(x, y)$. It is important to state this lemma without unnecessary restrictions such as $\left\{S_{i}\right\}_{i=1}^{m}$ being a set of wslices or $E$ being a curve, as these would limit its usefulness. For instance, the second restriction would invalidate the use of the summation estimate in the proof of Theorem 2.14 below, and either restriction would invalidate the use of a Euclidean version of it in [B, Proposition 3.3].

Corollary 2.9 also provides a rather simple method of producing domains that do not satisfy $\alpha$-wslice conditions. Suppose $\Gamma \subset \mathbb{R}^{n}$ is a domain and that $\Omega=G \backslash E$ is a subdomain of $G$ such that $E \cap K$ is a finite set for all compact subsets $K$ of $G$. Suppose now that $\left\{S_{i}\right\}_{i=1}^{m}$ is an $(\alpha, C)$-wslice dataset for the points $x, y \in \Omega$ with respect to the domain $\Omega$, and that $\delta_{\Omega}(z) \approx \delta_{G}(z)$ for $z=x$ and $z=y$. It is a routine exercise to show that each $S_{i}$ also satisfies (WS-1) for the same pair of points with respect to the domain $G$ (hint: make little bypasses around each point of $E$ that a path $\lambda$ passes through). Consequently, Corollary 2.9 tells us that

$$
\mathrm{WS}_{\alpha}(x, y ; \Omega ; C) \lesssim\left[\delta_{G}^{\alpha}(x)+\delta_{G}^{\alpha}(y)+d_{\alpha, G}(x, y)\right] .
$$

We can therefore produce a domain that fails to satisfy an $\alpha$-wslice condition and, by removing a countable family $E$ of points which force paths between the points $x, y$, go through enough bottlenecks to make $d_{\alpha, \Omega}(x, y)$ much larger than $\delta_{G}^{\alpha}(x)+$ $\delta_{G}^{\alpha}(y)+d_{\alpha, G}(x, y)$. For instance, the domain in Figure 2.6 is not a 0 -wslice domain for precisely this reason: if we take the points $x, y$ to have Euclidean coordinates $\left(3 \cdot 2^{-k}, 0\right)$ and $\left(3 \cdot 2^{-k-1}, 0\right)$, respectively, and let $k$ tend to infinity, then $d_{0, G}(x, y)$ remains bounded, but $d_{0, \Omega}(x, y)$ tends to infinity. The case $\alpha>0$ is different since $d_{\alpha, G}(x, y)$ and $d_{\alpha, \Omega}(x, y)$ are then both comparable to $2^{-k \alpha}$. 
Using a slight twist on the same idea, we see that the domain $\Omega^{\prime}$ mentioned above fails to be an $\alpha$-wslice domain for each $\alpha$. Let us consider only the case $\alpha>0$ and leave $\alpha=0$ as an exercise to the reader. Take the same pair of points $x, y$ as for the last example (or move them a little if either is not in $\Omega^{\prime}$ ), with $k$ being very large but fixed. Define the domain $G^{\prime}$ to be the triangle $G$ less two points, namely the closest points in $E^{\prime}$ to $x$ and $y$; if these are not unique, any choice will do. It is readily checked that $d_{\alpha, G^{\prime}}(x, y) \lesssim 2^{-k \alpha}$, whereas $d_{\alpha, \Omega^{\prime}}(x, y) \geq 2^{k !(1-\alpha)} \cdot 2^{-k \alpha}$. Using the previous estimates for the domains $G^{\prime}$ and $\Omega^{\prime}$, we get a contradiction to any given $(\alpha, C)$-wslice condition by picking a large enough value of $k$.

We finish this subsection by making a few more general comments on sets of wslices. Let us write $\gamma_{\mathcal{F}}=\gamma^{*} \cap\left(\bigcup_{S \in \mathcal{F}} S\right)$, whenever $\mathcal{F}$ is a set of wslices. To prove an $\alpha$-wslice condition for the pair $x, y \in \Omega$, it suffices to find a set of wslices $\mathcal{F}$ (and associated wnumbers) and an $\alpha$-efficient path $\gamma$ for $x, y$ such that $\operatorname{len}_{\alpha, \Omega}(\gamma) \leq$ $C_{3} \operatorname{len}_{\alpha, \Omega}\left(\gamma_{\mathcal{F}}\right)$, and (WS-4) holds for all $S_{i} \in \mathcal{F}$. Of course the associated constants $C_{i}, i=1, \ldots, m$, should be uniform over all pairs of points $x, y \in \Omega$. When (2.3) fails to prove a wslice condition, it is because (WS-4) fails for one or more of the associated slices which in turn is usually because (WS-4a) fails to hold. Roughly speaking, this indicates that these annuli are too "fat" and need to be replaced by a larger set of "thinner" slices, each of which covers less of the efficient path. Conversely, (WS-1) acts as a lower bound on how thin slices are allowed to be, and this lower bound is stricter in the inner wslice condition than in the Euclidean case. In the various examples of non-wslice domains above, (WS-1) forces most would-be slices to be so fat that (WS-4) and (WS-4a) both fail. It is worthwhile to look at the examples in Figures 2.6 and 2.7 with this idea in mind.

2.10. The extra hypotheses. It is convenient in what follows to use (WS- $N: C$ ) to refer to condition (WS- $N$ ) with a specified value of $C$. General wslice datasets do not have to be as simple and nice as the examples given previously, in which all the extra conditions (WS-4), (WS-4a), (WS-5) and (WS-1 ${ }^{+}$) clearly hold in each case. However, we see in this section that if there is any wslice dataset, there is also a "nice" wslice dataset that satisfies most of these extra conditions. Let us consider one simple way of making a "nice" dataset "nasty", as illustrated in Figure 2.11. Take two adjacent slices $S_{i}$ and $S_{i+1}$, cut them into a large number of subslices which are like the slices but much thinner (we ignore the boundaries between subslices, i.e. the dotted lines in the diagram), and now replace the two old slices $S_{i}$ and $S_{i+1}$ by two new slices $U$ and $V$ defined, respectively, as the unions of the odd and of the even subslices when listed in their natural order, as indicated in the diagram. Since $U, V$ both consist of many thin layers, we can destroy conditions (WS-5: $C^{\prime}$ ) and (WS- $1^{+}: C^{\prime}$ ) for any fixed $C^{\prime}$, while preserving the $\alpha$-wslice condition (perhaps with a somewhat larger $C$, but with a value independent of $C^{\prime}$ ) if we cut the original slices into a sufficiently large number of subslices. Of course these new slices are rather unnatural, and the original slices in the diagram do satisfy (WS-5) and $\left(\mathrm{WS}_{-1}{ }^{+}\right)$.

Similarly the extra conditions (WS-4), (WS-4a) fail for general wslice datasets. Consider the (nice) dataset used for the $\alpha=0$ proof of (2.3) and add to it an extra slice of the form $B(x, d(x, y) / 2) \backslash \bar{B}\left(y, \delta_{\Omega}(y) / 2\right)$ where $d(x, y)$ is much larger than $d(y)$ (and $d(y) \geq d(x))$. If $\alpha=0$, we can destroy (WS-4a: $C^{\prime}$ ) and (WS-4: $C^{\prime}$ ) for arbitrary $C^{\prime}$, while preserving the $(\alpha, C)$-wslice property of the dataset by making such a modification. The same example obviously works for (WS-4a) when $\alpha>0$, 


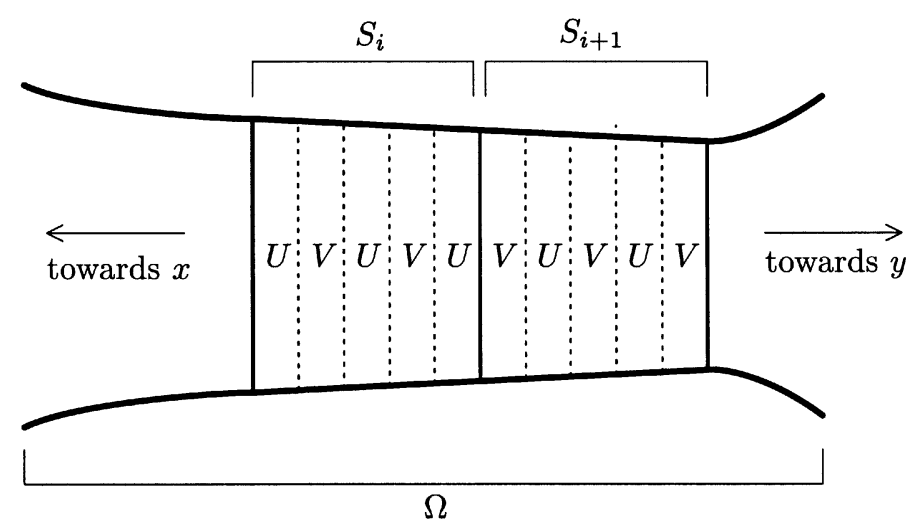

FiguRE 2.11. Slices and subslices

and (WS-4) for $\alpha>0$ is left to the reader. Note that if we simply remove the extra slice, we get "nice" $\alpha$-wslice datasets that satisfy all the extra conditions (WS-4), (WS-4a), (WS-5), and (WS-1 ${ }^{+}$).

Below we consider whether or not we can in general "fix up" a general $\alpha$-wslice dataset to get a nicer $\alpha$-wslice dataset that satisfies these extra conditions. One condition has already been examined: it is shown in [BS1, Lemma 2.3] that every (inner) $(\alpha, C)$-wslice domain has $\alpha$-wslice datasets that satisfy (WS-4). In fact any given wslice dataset for an arbitrary pair of points $x, y$ can be modified to obtain an $(\alpha, 4 C)$-wslice dataset for $x, y$ which satisfies (WS-4: $\left.C^{\prime}\right)$ for some $C^{\prime}=C^{\prime}(C, \alpha)$.

Below, we give a short proof of (WS-4) in our most general setting, and we prove (WS-4a) and (WS-5) for many metric spaces including all (inner) Euclidean domains; the path $\gamma$ in (WS-4) and (WS-4a) can be any sufficiently efficient path, but the dataset must be tailored to whatever path is chosen.

The proof of (WS-5) solves part of an open problem in [BS2, Section 6], where it was conjectured that (WS-5) and (WS- $1^{+}$) could be made to hold in an $\alpha$-wslice domain. We emphasize that BS2 was concerned only with Euclidean domains, while our investigations deal with more general metric spaces.

Lemma 2.12. If $\Omega$ is an $(\alpha, C)$-wslice space, then for every $x, y \in \Omega$ there is an $(\alpha, 4 C)$-wslice dataset for $x, y$ which satisfies (WS-4: 4C). Moreover, the dataset can be chosen to accommodate any $(\alpha, 1 / 4,0)$-efficient path $\gamma \in \Gamma_{\Omega}(x, y)$.

Lemma 2.13. If $\Omega$ is an $(\alpha, C)$-wslice space which is locally $C^{\prime}$-externally connected, then for every $x, y \in \Omega$ there is an $(\alpha, 8 C)$-wslice dataset for $x, y$ which satisfies (WS-4: $4 C$ ) and (WS-4a: $\left.C^{\prime \prime}\right)$ for some $C^{\prime \prime}=C^{\prime \prime}\left(\alpha, C, C^{\prime}\right)$. Moreover, the dataset can be chosen to accommodate any $(\alpha, 1 / 4,0)$-efficient path $\gamma \in \Gamma_{\Omega}(x, y)$.

Theorem 2.14. If $\Omega$ is an $(\alpha, C)$-wslice space which is locally $\left(C_{0}, C_{0}^{\prime}\right)$-nicely connected, then for every $x, y \in \Omega$ there is an $\left(\alpha, C^{\prime}\right)$-wslice dataset for $x, y$ which satisfies (WS-5: $C^{\prime}$ ) for some $C^{\prime}=C^{\prime}\left(\alpha, C, C_{0}, C_{0}^{\prime}\right)$.

Some restriction such as local nice connectivity is needed in order to prove (WS5). It is best, though, to put off a counterexample until the next section; see Example 3.5. 
There is one significant gap in what we can prove: we do not know if $\left(\mathrm{WS}-1^{+}\right.$) can be made to hold even in the Euclidean setting, although we would make the following conjecture.

Conjecture. If $(\Omega, d)$ is an $(\alpha, C)$-wslice space which is locally $\left(C_{0}, C_{0}^{\prime}\right)$-nicely connected, then it is also an $\left(\alpha, C_{1}\right)$-wslice ${ }^{+}$space, for some $C^{\prime}=C^{\prime}\left(\alpha, C, C_{0}, C_{0}^{\prime}\right)$.

Proof of Lemma 2.12. For $x, y \in \Omega$, we choose an arbitrary $(\alpha, 4 C / 3)$-wslice dataset $\left\{\left(S_{i}, d_{i}\right)\right\}_{i=1}^{m}$. We may assume that $d_{\alpha, \Omega}(x, y) \leq 2 C \sum_{i=1}^{m} d_{i}^{\alpha}$, since otherwise taking the empty set as our $(\sigma, 2 C)$ admissible set already gives an $(\alpha, 2 C)$-wslice condition for $x, y$, and so (WS-4) is trivially true.

Choose any $(\alpha, 1 / 4,0)$-efficient path $\gamma \in \Gamma_{\Omega}(x, y)$ and discard those slices $S_{i}$ that fail to satisfy (WS-4: $4 C$ ) with this choice of path $\gamma$. Define $I$ and $J$ to be the sets of non-discarded and discarded indices, respectively. Then

$$
4 C \sum_{j \in J} d_{j}^{\alpha} \leq \operatorname{len}_{\alpha, \Omega}(\gamma) \leq \frac{5}{4} \cdot 2 C \sum_{i=1}^{m} d_{i}^{\alpha} .
$$

Thus $\sum_{i \in I} d_{i}^{\alpha} \geq(3 / 8) \sum_{i=1}^{m} d_{i}^{\alpha}$, and the slimmed-down collection $\left\{S_{i}, d_{i}\right\}_{i \in I}$ serves as an $\left(\alpha, C^{\prime}\right)$-wslice dataset for $x, y$, where $C^{\prime}=(8 / 3) \cdot(4 C / 3)<4 C$.

Proof of Lemma 2.13. Fix any $(\alpha, 1 / 4,0)$-efficient path $\gamma \in \Gamma_{\Omega}(x, y)$, and apply Lemma 2.12 to obtain an $(\alpha, 4 C)$-wslice dataset which satisfies (WS-4: $4 C$ ) for $\gamma$, and let $\sigma_{i} \equiv \gamma^{*} \cap S_{i}$. The local external connectivity condition is required only for the upper bound on $\delta_{\Omega}(z)$ in (WS-4a), which follows from Lemma 2.8 (since for $C_{1}^{\prime} \leq C_{2}^{\prime}$, local $C_{1}^{\prime}$-external connectivity implies local $C_{2}^{\prime}$-external connectivity, we may replace $C^{\prime}$ by $C^{\prime} \vee 2$ to make Lemma 2.8 applicable).

It remains to establish the lower bound for $\delta_{\Omega}(z)$, for $z \in \sigma_{i}\left(=\gamma^{*} \cap S_{i}\right)$ in (WS4a). We set $E_{i}=\left\{z \in \sigma_{i}: \delta_{\Omega}(z) \leq c d_{i}\right\}$, where $c \equiv\left(32 C^{2}\right)^{-1 /(1-\alpha)}$. If $E_{i}=\emptyset$, the lower bound will hold (with $C^{\prime \prime}=c^{-1}$ ) so we assume that $E_{i} \neq \emptyset$. Using (WS-4: $4 C)$, we obtain $\operatorname{len}_{\alpha, \Omega}\left(E_{i}\right) \leq \operatorname{len}_{\alpha, \Omega}\left(\sigma_{i}\right) \leq 4 C d_{i}^{\alpha}$ which leads us to conclude that $\operatorname{len}_{d}\left(E_{i}\right) \leq \frac{4 C d_{i}^{\alpha}}{\left(c d_{i}\right)^{\alpha-1}}=\frac{d_{i}}{8 C}$. Thus we can remove $E_{i}$ from $S_{i}(1 \leq i \leq m)$ to get a new dataset which enjoys all of the asserted properties.

Proof of Theorem 2.14. We first augment $C$, if necessary, to assure that $C^{\alpha}>4$. Let $\gamma \in \Gamma_{\Omega}(x, y)$ be a path which is $(\alpha, 1 / 4,0)$ efficient for the points $x, y \in \Omega$. Invoking Lemma 2.13, we choose an $(\alpha, 8 C)$-wslice dataset $\left\{\left(S_{i}, d_{i}\right)\right\}_{i=1}^{m}$ for $x, y$ which satisfies (WS-4: $4 C$ ) and (WS-4a: $C^{\prime \prime}$ ) for the given path $\gamma$. As in the proof of the previous lemma, we may also assume that $C_{0}^{\prime} \geq 16 C$, and similarly we can arrange things so that $C^{\prime \prime} \geq C_{0}^{\prime}$. We also arrange the indexing so that the wnumbers $d_{i}$ are in decreasing order.

Letting $M_{i}=\sup _{z \in S_{i}} \delta_{\Omega}(z)$, it follows from (WS-1: $8 C$ ) that $\operatorname{len}_{\alpha, \Omega}\left(\gamma^{*} \cap S_{i}\right) \geq$ $M_{i}^{\alpha-1} d_{i} / 8 C$. Combining this with (WS-4: $4 C$ ), we deduce that

$$
M_{i} \geq(4 \sqrt{2} C)^{-2 /(1-\alpha)} d_{i}, \quad 1 \leq i \leq m .
$$

For each $i$, pick $z_{i} \in S_{i}$ with $\delta_{\Omega}\left(z_{i}\right) \geq M_{i} / 2$, and let $B_{i}=B_{d}\left(z_{i}, r_{i}\right)$, where $r_{i}=\delta_{\Omega}\left(z_{i}\right) / 2$. It is useful to note that $r_{i} \approx d_{i}(1 \leq i \leq m)$. In fact, by using (2.15) for one direction, and Lemma 2.8 and our assumption $C_{0}^{\prime} \geq 16 C$ for the other, we get

$$
\frac{d_{i}}{4(4 \sqrt{2} C)^{2 /(1-\alpha)}} \leq r_{i} \leq \frac{\left(C_{0}^{\prime}\right)^{2} d_{i}}{2}
$$


Let $I_{j}$ be the set of all indices $i$ such that $S_{i}$ intersects $B_{j}$ and $r_{i} / r_{j} \in(1 / 2,1]$. We shall show that $N_{j}$, the cardinality of $I_{j}$, is bounded by a number $M=$ $M\left(\alpha, C, C_{0}, C_{0}^{\prime}\right)$. Suppose $N_{j}>0$ and let $E_{j}=\bigcup_{i \in I_{j}} \gamma^{*} \cap \overline{S_{i}}$. By (2.16), we deduce that the slices $S_{i}, i \in I_{j}$, all fit inside the ball $B_{d}\left(z_{j}, C_{1} r_{j}\right)$ for some $C_{1} \lesssim 1$. We consider balls $\left\{B^{\prime}(u) \equiv B\left(u, r_{j} / 4 C_{0}\left(C_{0}^{\prime}\right)^{2} C^{\prime \prime}\right)\right\}$, where $u \in \overline{E_{j}}$, and take a maximal collection of them: $B_{1}^{\prime}=B^{\prime}\left(u_{1}\right), B_{2}^{\prime}=B^{\prime}\left(u_{2}\right), \cdots, B_{p}^{\prime}=B^{\prime}\left(u_{p}\right)$, with the property that $\left\{\frac{1}{3} B_{i}^{\prime}\right\}$ is a disjoint family. We arrange the indexing so that $u_{1}, u_{2}, \cdots, u_{p}$ proceed in the natural order induced by $\gamma$. Since all of the pairwise disjoint smaller balls have the same radius $r_{j} / 12 C C_{0} C_{0}^{\prime}\left(C^{\prime \prime}\right)^{2}$ and are contained in the single ball $B_{d}\left(z_{j},\left(C_{1}+1\right) r_{j}\right)$, we conclude that $p \lesssim 1$.

Claim. $B_{i}^{\prime} \subset B_{d_{\alpha, \Omega}}\left(u_{i},\left(C^{\prime \prime}\right)^{\alpha} d_{j}^{\alpha}\right)$.

Proof of Claim. Let $y \in B_{i}^{\prime}$, so that $d\left(u_{i}, y\right)<r_{j} / 4 C_{0}\left(C_{0}^{\prime}\right)^{2} C^{\prime \prime}$. Now, since $u_{i} \in$ $S_{i^{\prime}}$ for some $i^{\prime} \in I_{j}$, (WS-4a: $\left.C^{\prime \prime}\right)$ and (2.16) give us that $\delta_{\Omega}\left(u_{i}\right) \geq d_{i^{\prime}} / C^{\prime \prime} \geq$ $r_{i^{\prime}} /\left(C_{0}^{\prime}\right)^{2} C^{\prime \prime} \geq r_{j} / 2\left(C_{0}^{\prime}\right)^{2} C^{\prime \prime}$. In particular, $d\left(u_{i}, y\right)<\delta_{\Omega}\left(u_{i}\right) / 2 C_{0}$. Since $y \in$ $C_{0}^{-1} B_{u_{i}}$, local quasiconvexity implies that $y$ and $u_{i}$ can be connected by a path $\tau$ of length at most $C_{0} d\left(u_{i}, y\right)<\delta_{\Omega}\left(u_{i}\right) / 2$. Thus for all $w \in \tau^{*}, \delta_{\Omega}(w) \geq \delta_{\Omega}\left(u_{i}\right) / 2$, and hence $\operatorname{len}_{\alpha, \Omega}(\tau)<\left[\delta_{\Omega}\left(u_{i}\right) / 2\right]^{1+(\alpha-1)}$. Now $\delta_{\Omega}\left(u_{i}\right) \leq C^{\prime \prime} d_{i^{\prime}} \leq 2 C^{\prime \prime} d_{j}$, and the claim follows.

In light of the claim, we can modify $\gamma$ by replacing (in order) the portions of it which intersect the balls $B_{1}^{\prime}, B_{2}^{\prime}, \cdots, B_{p}^{\prime}$ by $(\alpha, 1,0)$-efficient paths from the first entry point to the last exit point with the proviso that if one of these balls is not disjoint from a preceding one, then the starting point for this (closed) ball will be the last intersection point for the immediately preceding (closed) ball. We thus get a new path (parametrized by arclength, say) $\lambda \in \Gamma_{\Omega}(x, y)$. Applying the mutual comparability of $r_{i}, i \in I_{i}$, followed in order by Corollary 2.9 with $E=\bigcup_{i \in I_{j}} \lambda^{*} \cap S_{i}$, our claim, and the boundedness of $p$, we see that

$$
N_{j} d_{j}^{\alpha} \approx \sum_{i \in I_{j}} d_{i}^{\alpha} \lesssim \operatorname{len}_{\alpha, \Omega}\left(E_{j}\right) \lesssim p d_{j}^{\alpha} \lesssim d_{j}^{\alpha}
$$

Thus $N_{j}$ is bounded as desired.

More generally, there are at most some bounded number, $M^{\prime}=M^{\prime}\left(\alpha, C, C_{0}, C_{0}^{\prime}\right)$, of slices $S_{i}$ that intersect $B_{j}$ and for which $d_{i}<d_{j}$. To see this we can use the above argument to get a bound on the number of such slices that satisfy a restriction of the form $r_{i} / r_{j} \in\left(2^{l-1}, 2^{l}\right]$ for each integer $l$. This bound will depend on $l$, but this is not a problem since there can be no such slices unless $|l| \lesssim 1$. The upper bound on $l$ follows from the fact that $d_{i}<d_{j}$ and $r_{i} \approx d_{i}$. By (2.16), it suffices to prove the lower bound when $d_{i}<r_{j} / 2$. In this case, $S_{i} \subset B\left(z_{j}, 3 r_{j} / 2\right)$, and so $\delta_{\Omega}(z) \geq \delta_{\Omega}\left(z_{j}\right) / 4$ for all $z \in S_{i}$. Fixing $z \in \gamma^{*} \cap S_{i}$, and using (WS-4) and (2.16) again, we see that $C^{\prime \prime} d_{i} \geq \delta_{\Omega}(z) \geq r_{j} / 2 \gtrsim d_{j}$, which gives the required lower bound.

Defining $S_{i}^{\prime} \equiv S_{i} \cup B_{i}$, we next partition the integers from 1 to $m$ into sets $I$ and $R$ (included and rejected indices); the desired dataset will be $\left\{S_{i}^{\prime}, d_{i}\right\}_{i \in I}$. We allocate 1 to $I$. Inductively, we allocate $i$ to $R$ if $S_{i}$ intersects $B_{j}$ for some $j<i, j \in I$, and otherwise we allocate it to $I$. Recalling that the $d_{i}^{\prime} s$ are nonincreasing, it follows that $\sum_{i=1}^{m} d_{i}^{\alpha} \leq M^{\prime} \sum_{i \in I} d_{i}^{\alpha}$, and hence the new collection serves as an $\left(\alpha, 8 C M^{\prime}\right)$-wslice dataset for $x, y$. Since the slice $S_{i}^{\prime}$ contains a ball of radius $r_{i}=\delta_{\Omega}\left(z_{i}\right) / 2 \geq M_{i} / 4,(2.15)$ now shows the validity of (WS-5: $C^{\prime}$ ) with $C^{\prime}=(8 \sqrt{2} C)^{2 /(1-\alpha)}$. 
One way to prove that any $\alpha$-wslice condition fails is to get an upper bound on the number of slices. A good way of doing this is to have two paths between the same endpoints that are mostly rather far apart, using (WS-1) to bound the number of slices that cover various parts of the first path that lie at various distances from the second. The next lemma involves a rather abstract form of this argument; it is rather similar to Corollary 2.9, but the set $A$ is measured using a different length measure.

Lemma 2.17. Suppose $(\Omega, d)$ is a rectifiably connected metric space, $A \subset \Omega$ is a rectifiable set and, for some $c>0, \mathcal{F}$ is a collection of disjoint non-empty bounded subsets of $\Omega$ with $\operatorname{len}_{d}(S \cap A) \geq c d_{S}$ for all $S \in \mathcal{F}$. Suppose also that there is a constant $C$ and a function $g: A \rightarrow(0, \infty)$ such that $d_{S} \geq g(x)$ whenever $x \in S \cap A$, and $\operatorname{len}_{d}\left(g^{-1}(0, t)\right) \leq C t$ for all $t>0$. Then, for each $0<\alpha<1$, we have

$$
\sum_{S \in \mathcal{F}} d_{S}^{\alpha} \leq \frac{2^{1+\alpha} C[\operatorname{len}(A)]^{\alpha}}{c^{1+\alpha}\left(1-2^{-\alpha}\right)}
$$

If, additionally, $g(x)>\epsilon>0$ for all $x \in A$, then the cardinality of $\mathcal{F}$ is at most $2 C c^{-1} \log _{2}(4 \operatorname{len}(A) / c \epsilon)$.

Proof. We partition $\mathcal{F}$ into subsets $\mathcal{F}_{k}, k \in \mathbb{Z}$, by the rule $S \in \mathcal{F}_{k}$ if $d_{S} \in\left(2^{k-1}, 2^{k}\right]$. Note that if $S \in \mathcal{F}_{k}$, then $\operatorname{len}_{d}(S \cap A)>c 2^{k-1}, S \cap A \subset g^{-1}\left(\left(0,2^{k}\right]\right)$, and $\operatorname{len}\left(g^{-1}\left(\left(0,2^{k}\right]\right)\right) \leq C 2^{k} \wedge \operatorname{len}(A)$. It follows that the cardinality of each $\mathcal{F}_{k}$ is at most $2 C / c$ and that $\mathcal{F}_{k}$ is empty whenever $2^{k}>2 \operatorname{len}(A) / c$. Thus $\sum_{S \in \mathcal{F}_{k}} d_{S}^{\alpha}$ is at most $2 C c^{-1} 2^{k \alpha}$ and, summing over allowable values of $k$, the desired estimate follows. The last statement is similar but easier, since we are just summing subset cardinalities bounded by $2 C c^{-1}$ for each value of $k$ satisfying $\epsilon<2^{k} \leq 2 \operatorname{len}(A) / c$.

\section{WEAK SLICE BUT NOT INNER WEAK SLICE}

In this section we construct examples of $\alpha$-wslice domains which are not inner $\alpha$-wslice domains thereby resolving Open Problem D in [BS2]. The pathology of such examples is most easily understood in the setting of manifolds imbedded in Euclidean space and we will precede the domain example with a corresponding manifold example. Note that, since the ambient Euclidean metric is dominated by the inner metric on a manifold, and the length of a path on the manifold is the same for both metrics, it is clear that inner $\alpha$-wslice manifolds are $\alpha$-wslice manifolds.

We begin with an example of a 2-manifold in $\mathbb{R}^{3}$. Since the example is independent of the parameter $\alpha$, it will show that the inner wslice conditions can be much more restrictive than wslice conditions. The requisite pathological property becomes more apparent in this flexible manifold setting. Subsequently, when we construct a (flat) domain in $\mathbb{R}^{2}$ with similar properties, the construction will require some modifications to work around the flatness. In any case, after the groundwork is laid for the manifold example it will be a lot less work to indicate the changes needed to verify the domain example, and the careful reader should be able to glean the common phenomenon which occurs in both examples.

Example 3.1. We construct a 2-manifold $M \subset \mathbb{R}^{3}$ which is an $\alpha$-wslice manifold for every $\alpha \in[0,1)$, but satisfies none of the corresponding inner $\alpha$-wslice conditions.

The 2-manifold $M$, whose construction depends on a decreasing sequence of positive parameters $\left\{\delta_{j}\right\}$ (which we shall specify later) is illustrated in Figure 3.2. 


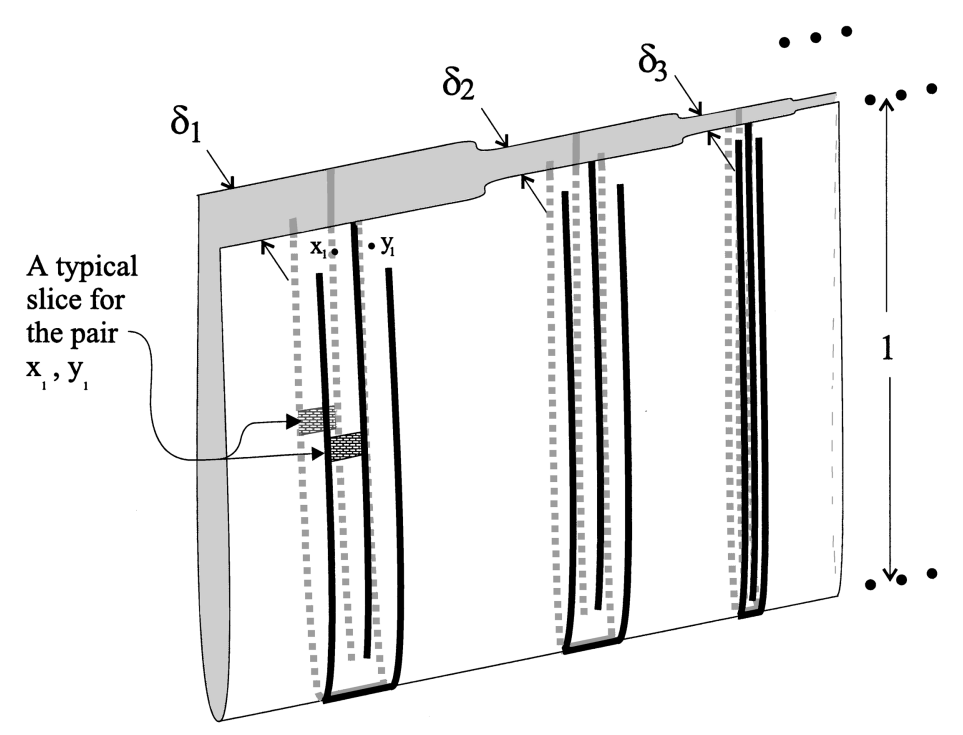

Figure 3.2. An illustration of the 2-manifold $M$ with a typical slice shown

It is helpful to first define another manifold $M^{\prime}$ by (smoothly) folding a rectangular sheet along a central axis of length $L \equiv \sum_{j=1}^{\infty} 1 /(j+1)^{2}$ in such a way that along the first $1 / 2^{2}=1 / 4$ length of the fold, a distance of approximately $\delta_{1}$ is maintained between the two folds (except near the bottom where the folding is done). On the next $1 / 3^{2}=1 / 9$ length of the fold, a distance of approximately $\delta_{2}$ is maintained, and so on. It is useful to coordinatize $M^{\prime}$ using the natural map $\phi$ to the original rectangular sheet. Thus, $\phi=\left(\phi_{1}, \phi_{2}\right)$ maps $M^{\prime}$ to $(-1,1) \times(0, L)$, and it maps the folding axis isometrically to $\{0\} \times(0, L)$. The point with coordinates $(x, y)$ lies approximately a distance $|x|$ above the folding axis point with coordinates $(0, y)$, and a distance $1-|x|$ from the rim of $M^{\prime}$, with positive values of $x$ corresponding to the front fold and negative values to the back fold. For this example only, whenever we give coordinates of the form $(a, b)$, these are meant to be $\phi$-coordinates of a point in $M^{\prime}$, not Euclidean coordinates.

We now define a submanifold $M$ of $M^{\prime}$ by taking the complement of an infinite collection of closed slits. Let us describe how these slits are made. At the center of the $j$ th portion of the horizontal folding axis (corresponding to a length of $1 /(j+1)^{2}$ ) a slit along the folding axis of length $2 \delta_{j}$ is made, between points $\left(0, a_{j}\right)$ and $\left(0, b_{j}\right)$, $b_{j}=a_{j}+2 \delta_{j}$; we also define $b_{0}=0$ for convenience. We write $I_{j} \equiv\left[a_{j}, b_{j}\right]$. We assume that $0<\delta_{j}<1 / 4(j+1)^{2}$ and that the numbers $\delta_{j}$ are monotonic decreasing. At the ends of each such slit, front and back vertical cuts are made producing slits from points $\left( \pm\left(1-\delta_{j}\right), a_{j}\right)$ to $\left(0, a_{j}\right)$, and from $\left( \pm\left(1-\delta_{j}\right), b_{j}\right)$ to $\left(0, b_{j}\right)$. Finally, midway between these two vertical slits, two more vertical slits are made, one on each fold. These slits start at $\left( \pm 1,\left(a_{j}+b_{j}\right) / 2\right)$ and end at $\left( \pm \delta_{j},\left(a_{j}+b_{j}\right) / 2\right)$. Although these slits are illustrated in Figure 3.2 by heavy thick black curves, this is merely to emphasize their presence. They are smooth curves with no width.

Step 1: We show that $M$ is a 0-wslice domain. Let us designate the $j$ th portion of $M$ to be the part of $M$ (of horizontal projection length $\left.1 /(j+1)^{2}\right)$ corresponding 
to the parameter $\delta_{j}$. We also designate the $j$ th network of slits to be those slits of $M$ which lie in its $j$ th portion. The $j$ th slit zone $Z_{j}$ is the set of all points $z \in M$ with $\phi_{2}(z) \in I_{j}$.

Let $z=\left(z_{1}, z_{2}\right)$ and $w=\left(w_{1}, w_{2}\right)$ be the points in $M$ for which we wish to establish a 0 -wslice condition, and let $\gamma$ be a 0 -geodesic for $z, w$. Without loss of generality, we assume that $\phi_{2}(z) \leq \phi_{2}(w)$. If neither $z$ nor $w$ lie in a slit zone, and the interval $\left[z_{2}, w_{2}\right]$ intersects no interval $I_{j}$, then $z$ and $w$ can be joined by a uniform path. Putting together (1.1) and (2.3), the wslice condition follows. We may assume therefore that $\left[z_{2}, w_{2}\right]$ intersects at least one interval $I_{j}$.

Let us examine the case where $\left[z_{2}, w_{2}\right]$ contains the intervals $I_{j}$ that it intersects, namely $j_{1} \leq j \leq j_{2}$. The other case, in which either $z$ or $w$ lies in a slice zone will be handled in a similar fashion, so we leave it to the reader. Suppose $\gamma$ has an initial segment (beginning at $z$ ) on which distance to the boundary is at most $\delta_{j_{1}} / 4$, or a final segment (ending at $w$ ) on which distance to the boundary is at most $\delta_{j_{2}} / 4$. We discard both these segments if they exist and call the truncated path $\nu$. We may assume that the quasihyperbolic length of $\nu$ is at least half that of $\gamma$, since otherwise it is easily verified that the wslice condition follows from (1.1) and (2.3) (since the discarded segments move more or less "directly away from the boundary").

Let $E_{j}$ be the part of $\nu^{*}$ that lies in $\phi_{2}^{-1}\left(\left[b_{j-1}, a_{j}\right]\right)$, and let $F_{j}$ be the part of $\nu^{*}$ that lies in $\phi_{2}^{-1}\left(\left[a_{j}, b_{j}\right]\right)$. It is easy to check that $\delta_{M}(x) \gtrsim \delta_{j}, x \in E_{j}$, and that $\operatorname{len}_{0, M}\left(E_{j}\right) \lesssim \log \left(1 / \delta_{j}\right)$. When $j$ is large, this length is much less than $\operatorname{len}_{0, M}\left(F_{j}\right) \approx 1 / \delta_{j}$. Note that the lower bound implicit in this last estimate is rather easy to establish, while for the upper bound we use the fact that $\delta_{M}(x) \gtrsim \delta_{j}$, $x \in E_{j} \cap F_{j}$ (and hence for all $x \in F_{j}$ ). It suffices therefore to find a sufficiently numerous set of 0 -wslices which cover most of the quasihyperbolic length of $F_{j} \cap \nu^{*}$, $j_{1} \leq j \leq j_{2}$.

Within the slice networks, we use as slices approximately square boxes (actually two of them for each slice, opposite one another on the two folds as depicted in Figure 3.2); we do this from a distance $\delta_{j}$ above the fold until we reach a distance $\delta_{j}$ or less from the top of the outer slits, and take their diameters as the associated 0 -wnumbers. With respect to the ambient Euclidean metric of $\mathbb{R}^{3}$, these slices have diameter approximately $\delta_{j}$ and certainly satisfy (WS-1). Indeed, for this condition a moderate value of $C$ suffices (e.g., $C=\sqrt{3}$ would work if the front-to-back distance is exactly $d_{j}$ and the $\delta_{j}$ 's are taken sufficiently small in the construction of $M$ ). Condition (WS-2) is also clear. Assuming as we may that the height of each fold is about one, the number of such slices within the $j$ th slit network is $0 \vee\left\lceil 2 \delta_{j}^{-1}-6\right\rceil$, thus giving (WS-3) with a moderate value of $C$ which is independent of the particular values of the $\delta_{j}$ 's.

Step 2: We show that the manifold $M$ cannot satisfy an inner 0-wslice condition. We consider pairs of points: $x_{j}, y_{j}$ which are situated with respect to the same (front) fold of the $j$ th slice network as are the points $x_{1}, y_{1}$ with respect to the first slice network, as illustrated in Figure 3.2. Thus $x_{j}=\left(1-\delta_{j},\left(3 a_{j}+b_{j}\right) / 4\right)$ and $y_{j}=\left(1-\delta_{j},\left(3 b_{j}+a_{j}\right) / 4\right)$. We shall show that, no matter what the value of $C$, the $(0, C)$-wslice condition fails for the pair $x_{j}, y_{j}$ when $j$ is sufficiently large.

We define two paths $\lambda_{j}, \nu_{j} \in \Gamma_{M}\left(x_{j}, y_{j}\right)$, both parametrized by arclength. Both are made up of only horizontal and vertical linear segments (with respect to our coordinate system). First $\lambda_{j}$ corresponds in our coordinate system to a concatenation 
of three line segments from $x_{j}$ to $\left(\delta_{j} / 2,\left(3 a_{j}+b_{j}\right) / 4\right)$, then to $\left(\delta_{j} / 2,\left(3 b_{j}+a_{j}\right) / 4\right)$, and finally to $y_{j}$; this is clearly a 0 -efficient path. The second path $\nu_{j}$ is more complicated: it goes down the front fold to the left of the $j$ th slit zone, then up the back fold, down and up the back slit network, and finally down the back fold to the right of the $j$ th slit zone and up the front to $y_{j}$. Specifically, we define $\nu_{j}$ to correspond in our coordinate system to a concatenation of nine line segments that join the following points in sequence:

$$
\begin{array}{lll}
x_{j}=\left(1-\delta_{j} / 2,\left(3 a_{j}+b_{j}\right) / 4\right), & \left(1-\delta_{j} / 2, a_{j}-\delta_{j} / 2\right), & \left(-1+\delta_{j} / 2, a_{j}-\delta_{j} / 2\right), \\
\left(-1+\delta_{j} / 2,\left(3 a_{j}+b_{j}\right) / 4\right), & \left(-\delta_{j} / 2,\left(3 a_{j}+b_{j}\right) / 4\right), & \left(-\delta_{j} / 2,\left(3 b_{j}+a_{j}\right) / 4\right), \\
\left(-1+\delta_{j} / 2,\left(3 b_{j}+a_{j}\right) / 4\right), & \left(-1+\delta_{j} / 2, b_{j}+\delta_{j} / 2\right), & \left(1-\delta_{j} / 2, b_{j}+\delta_{j} / 2\right), \\
y_{j}=\left(1-\delta_{j} / 2,\left(3 b_{j}+a_{j}\right) / 4\right) . & &
\end{array}
$$

It is easy to see that the portion of $A \equiv \lambda_{j}^{*} \backslash\left[C^{-1} B_{x_{j}} \cup C^{-1} B_{y_{j}}\right]$ that is within an inner distance $t$ of $\nu_{j}$ has length at most comparable with $t$, and is empty unless $t \gtrsim \delta_{j}$. In view of (WS-1), we can therefore apply Lemma 2.17 (Case $\alpha=0$ ) to deduce that the number of 0 -wslices is at most a bounded multiple of $\log 1 / \delta_{j}$. When $j$ is large, this is much smaller than $k_{M}\left(x_{j}, y_{j}\right) \approx 1 / \delta_{j}$, so every $(0, C)$-wslice condition must fail.

Final step: We show that the manifold $M$ satisfies the $\alpha$-wslice condition but not the inner $\alpha$-wslice condition whenever $\alpha \in(0,1)$. The changes required to adjust the case $\alpha=0$ proofs to handle the case $\alpha>0$ are all quite routine. In particular, for the positive result, the slice data constructions presented in Step 1 will work in this case as well, although the technical details will be somewhat different. The negative result is also quite similar. Note that the final appeal to Lemma 2.17 yields $\mathrm{WS}_{\alpha}\left(x_{j}, y_{j} ; M ; C\right) \lesssim 1$, whereas $d_{\alpha, M}\left(x_{j}, y_{j}\right) \approx \delta_{j}^{\alpha-1}$ is much larger for large $j$.

Note that the Euclidean metric on the manifold $M$ above is locally externally connected, but is not locally quasiconvex. Thus all of the theory in Section 2 is applicable to it with the exception of Theorem 2.14. However, it is easy to see that (WS-5) can be made to hold always in spite of this. The inner metric on $M$ is locally nicely connected, as is readily verified. In some ways, the Euclidean metric is somewhat unnatural on $M$ and so it certainly seems appropriate to give also a planar example $\Omega$ of the same phenomenon, as we now do.

In our subsequent domain construction we will work with some similarity transformations; so, for future reference we observe here some basic change of variable estimates. If $\sigma: \Omega \rightarrow \Omega^{\prime}$ is a similarity transform, i.e., $\sigma(x)=\mu x+x_{0}$, where $\mu>0$ is the similarity factor, we then have: $\delta_{\Omega^{\prime}}(\sigma(x))=\mu \delta_{\Omega}(x), \operatorname{len}_{\alpha, \Omega^{\prime}}(\sigma \circ \gamma)=$ $\mu^{\alpha} \operatorname{len}_{\alpha, \Omega}(\gamma)$, and thus $d_{\alpha, \Omega^{\prime}}(\sigma(x), \sigma(y))=\mu^{\alpha} d_{\alpha, \Omega}(x, y)$. From these relations, it now follows that all of the $\alpha$-wslice conditions are invariant under similarities.

Example 3.3. We construct a domain $\Omega \subset \mathbb{R}^{2}$ which, for every $\alpha \in[0,1)$, is an $\alpha$-wslice domain, but not an inner $\alpha$-wslice domain.

The domain $\Omega$ consists of the unit square $S$ in $\mathbb{R}^{2}$ with a sequence of rectangular slit domains $D_{j}(j \geq 2)$ separately attached along the top edge of $S$. The rectangular edges of $D_{j}$ have heights of $2^{-j}$ and widths of $4^{-j}$. To describe the slits removed from the corresponding rectangle $R_{j}$, it is simpler to describe them for the similar rectangle $R_{j}^{\prime}$ having vertices at $(-2,0),(2,0),\left(-2,2^{j+2}\right)$, and $\left(2,2^{j+2}\right)$. Note that there is a similarity mapping $\sigma_{j}$ from $R_{j}$ onto $R_{j}^{\prime}$ preserving top sides and with similarity factor $\mu_{j}=4^{j+1}$. The first slit in $R_{j}^{\prime}$ is along the midline from $\left(0,2^{j+2}\right)$ 


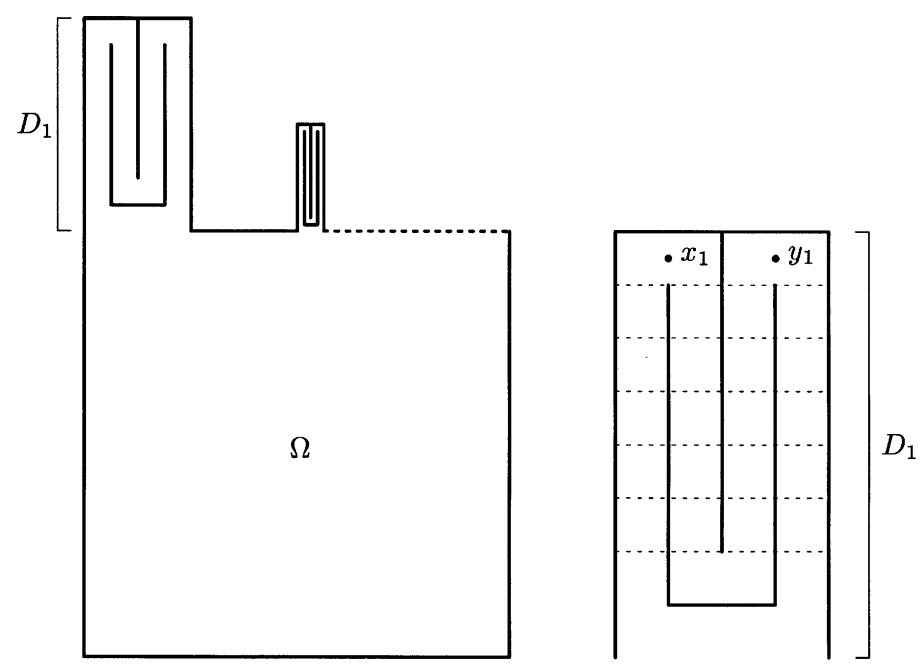

Figure 3.4. The domain $\Omega$ (left) and a magnified $D_{1}$ (right)

down to $(0,2)$. The other three slits form a U-shape from $\left(-1,2^{j+2}-1\right)$ down to $(-1,1)$, then right to $(1,1)$, and finally up to $\left(1,2^{j+2}-1\right)$. To show that the resulting domain $\Omega$ has the desired properties, many of the details of the manifold example can be applied here, so we outline only the main changes.

Consider the case $x=x_{j}, y=y_{j}$, where $x_{j}, y_{j} \in D_{j}$ are defined by the equations $x_{j}^{\prime}=\sigma_{j}\left(x_{j}\right)=\left(-1,2^{j+2}-.5\right)$ and $y_{j}^{\prime}=\sigma_{j}\left(y_{j}\right)=\left(1,2^{j+2}-.5\right)$. As slices $S_{i}$, we use pullbacks under $\sigma_{j}$ of the horizontal strips $S_{i}^{\prime}$ from $y=i+1$ to $y=i+2$ in $D_{j}^{\prime}=\sigma\left(D_{j}\right)\left(1 \leq i<2^{j+2}-2\right)$, and we write $d_{i}=\operatorname{dia}\left(S_{i}\right)$; the case $j=1$ is illustrated in Figure 3.4 (right), with slices bounded by dotted lines. Conditions (WS-1) and (WS-2) are easily verified. Since $k_{\Omega}\left(x_{j}, y_{j}\right)$ is approximately $2^{j}$, a 0 -wslice condition follows.

For other pairs of points $x, y$ lying in $D_{j}$, we might have to discard some slices, as (WS-1) will hold for less of them if they are quasihyperbolically closer together than our original pair, and (WS-2) might not hold for at most four of them (namely, any $S_{i}$ containing, or within a distance $4^{-j-1}$ of, $x$ or $y$ ). But as the reader can verify, there are enough of them that satisfy (WS-1) and (WS-2) in order to deduce a 0 -wslice condition with a moderate constant (uniform in $j$ ) unless the distance from either $x$ or $y$ to $\partial \Omega$ is smaller than $4^{-j-2}$. In this case, we supplement the set of wslices with some annular slices around the point that is closest to the boundary. The case of pairs of points situated in different $D_{j}$ 's, or anywhere else in $\Omega$ is left to the reader, as is the verification of an $\alpha$-wslice condition for $\alpha>0$.

The proof that $\Omega$ is not an inner $\alpha$-wslice domain for any $\alpha>0$ is very similar to that used in the manifold example. Suffice it to say that we consider the same points $x_{j}, y_{j}$ as in the second to last paragraph and take paths $\lambda_{j}, \nu_{j}$ that connect them inside $D_{j}$, where $\lambda_{j}$ consists of a few line segments that mainly travel down and up the "inner passage", while $\nu_{j}$ is similar but mainly travels down and up the "outer passage". We leave the details to the reader. 
Example 3.5. We construct a 2 -manifold $M \subset \mathbb{R}^{3}$ which, for every $\alpha \in[0,1)$, is an $\alpha$-wslice manifold for which the condition (WS-5) cannot be added.

We begin with a planar domain

$$
G=(0,1)^{2} \cup\left(\bigcup_{j=2}^{\infty} A_{j}\right),
$$

where $A_{j}=\left(a_{j}-\epsilon_{j}, a_{j}+\epsilon_{j}\right) \times\left(-b_{j}, 0\right], a_{j}=2^{-j}, b_{j}=2^{-2 j}$, and $\epsilon_{j}=2^{-2 j^{2}}$. This domain is simply connected, so certainly it satisfies an (inner) slice condition, and hence an (inner) $\alpha$-wslice condition for each $\alpha \in[0,1)$. We now map $G$ to a 2-manifold $M \subset \mathbb{R}^{3}$ via a bijective "folding map" $f$. Let us coordinatize $M$ using the natural $\mathbb{R}^{3}$ coordinates. We choose $f$ so that $f(x, y)=(x, y, 0)$ for all $(x, y) \in(0,1)^{2}$, and such that $f\left(A_{j}\right) \subset\left(a_{j}-\epsilon_{j}, a_{j}+\epsilon_{j}\right) \times\left(-\epsilon_{j}, 0\right] \times\left(-\epsilon_{j}, 0\right]$ by tightly folding the appendages $A_{j}$. Moreover we choose this folding map so that $f$ is a local quasi-isometry with respect to the Euclidean metrics on $G$ and $M$ (with, let's say, distortion factor at most 2), and an isometry with respect to the inner

metrics. Since $f$ is an inner isometry, $M$ is an inner $\alpha$-wslice manifold, and hence an $\alpha$-wslice manifold.

However, no matter how large a value of $C$ we choose, if we take $j=j(\alpha, C) \in$ $\mathbb{N}$ large enough, then every $(\alpha, C)$-wslice dataset $\left\{S_{i}, d_{i}\right\}_{i=1}^{m}$ for the points $z_{0}=$ $(1 / 2,1 / 2,0)$ and $z_{j}=f\left(a_{j},-b_{j}+\epsilon_{j}\right)$ fails to satisfy (WS-5). We sketch the proof of this when $\alpha=0$, leaving the $\alpha>0$ case to the reader. Let us define $E_{j}$ to be the $\epsilon_{j}$-neighbourhood of $f\left(A_{j}\right)$ with respect to the Euclidean metric. By going from $z_{0}$ to $z_{j}$ via two paths that stay far apart until they get near $f\left(A_{j}\right)$, one can prove as before (using Lemma 2.17) that the number of slices that are not contained in $E_{j}$ is at most comparable to $\log \epsilon_{j} \approx j^{2}$. On the other hand, $k_{M}\left(z_{0}, z_{j}\right) \approx b_{j} / \epsilon_{j}$ is much larger than this when $j$ is large, so there must be many more slices contained in $E_{j}$. All these other slices must have diameter comparable to $\epsilon_{j}$, and now (WS-5) is untenable since only a bounded number of disjoint balls of radius comparable to $\epsilon_{j}$ can be fit into the set $E_{j}$ whose diameter is comparable with $\epsilon_{j}$.

\section{The MEAN CigAR CONDitions}

For a constant $C \geq 1$, we say that a domain $\Omega \subsetneq \mathbb{R}^{n}$ is a $C$-uniform domain or inner $C$-uniform domain, if it is a $C$-uniform space when equipped with the Euclidean metric or inner Euclidean metric, respectively. Uniform domains include all bounded Lipschitz domains, as well as some domains with fractal boundary, such as the interior of a von Koch snowflake. All uniform domains are inner uniform, and a slit disk is a standard example of an inner uniform domain that is not uniform. For more on uniform and inner uniform domains, see [V3] and [V5].

Next, if $0 \leq \alpha \leq 1 \leq C$, we say that a rectifiably connected metric space $(\Omega, d)$ is an $(\alpha, C)$-mcigar space if for every pair $x, y \in \Omega$, there is an $(\alpha, C)$-mcigar path, i.e., a path $\gamma \in \Gamma_{\Omega}(x, y)$ such that:

$$
\operatorname{len}_{\alpha, \Omega}(\gamma) \leq \begin{cases}C d(x, y)^{\alpha}, & 0<\alpha \leq 1, \\ C \log \left(1+\frac{d(x, y)}{\delta_{\Omega}(x) \wedge \delta_{\Omega}(y)}\right), & \alpha=0 .\end{cases}
$$

We say that a domain $\Omega \subsetneq \mathbb{R}^{n}$ is an $(\alpha, C)$-mcigar domain or inner $(\alpha, C)$-mcigar domain, if it is an $(\alpha, C)$-mcigar space when equipped with the Euclidean metric 
or inner Euclidean metric, respectively. We note that in the Euclidean or inner Euclidean contexts, $\alpha$-mcigar conditions for $0<\alpha<1$ imply the existence of a path $\lambda$ that satisfies a type of cigar condition on average; see [BK2, Lemma 2.2] and Lemma 4.6 in [BS2].

Uniform domains are $\alpha$-mcigar domains for all $\alpha$. Gehring and Osgood GO showed that the classes of 0-mcigar domains and uniform domains coincide, and Väisälä [V5, 2.33] showed that the classes of inner 0-mcigar and inner uniform domains coincide. The class of (inner) $\alpha^{\prime}$-mcigar domains includes the class of (inner) $\alpha$-mcigar domains if and only if $\alpha \leq \alpha^{\prime}$. The Euclidean version is dealt with in $[\mathrm{L}$ and BK2; inclusion follows similarly in the inner case and the counterexamples in $[\mathrm{L}$ also handle the inner version. Thus mcigar domains include domains with rough (even fractal) boundary. Note that the class of inner uniform and inner mcigar domains contain their Euclidean analogues (strictly, since a planar slit disk is in all of the inner classes, but none of the Euclidean classes).

We refer the reader to BK2, GM1, and $\mathrm{D}$ for more information about $\alpha$ mcigar domains; these domains are called "weak cigar domains" in [BK2] and "Lip extension domains" in GM1] and [L] when $\alpha>0$. The last name derives from the fact that for $\alpha>0, G$ is $\alpha$-mcigar if and only if all functions which are locally Lipschitz of order $\alpha$ on $G$ are Lipschitz of order $\alpha$ on $G$; see [GM1].

We are now in a position to state the result mentioned in the introduction which provides an extremely large class of domains which satisfy all of the weak slice conditions of Section 2; this result is merely a restatement of Theorem 3.1 of [BS2]. As the result indicates, the large class of domains given even satisfy the stronger (inner) slice condition. Since we shall not use the slice condition, we choose to forgo its more complicated definition and refer the interested reader to [BK2] (see also BS1 or BS2).

Theorem 4.1. Let $\alpha \in[0,1)$ and let $f$ be a $K$-quasiconformal mapping from an inner $C$-uniform domain $G \subset \mathbb{R}^{n}$ onto $\Omega$. Then $\Omega$ is an inner $C^{\prime}$-slice domain and an inner $\left(\alpha, C^{\prime}\right)$-wslice $e^{+}$domain for some $C^{\prime}=C^{\prime}(C, n, K, \alpha)$.

\section{AN EXAMPLE INDICATING A BIG DIFFERENCE IN $\alpha$-MCIGAR DOMAINS: $\alpha=0$ vs. $\alpha>0$ IN RELATION TO WEAK SLICE CONDITIONS}

As Theorem 4.1 indicates, every quasiconformal image of an inner 0-mcigar domain is an inner slice domain and an inner $\alpha$-wslice ${ }^{+}$domain for all $\alpha \in[0,1)$. The related Theorem 3.6 of [BS2] says that if a broad domain is either an inner slice domain or an inner 0-wslice ${ }^{+}$domain, then its quasiconformal image is also an inner slice domain or an inner 0 -wslice ${ }^{+}$domain, respectively. In this section, we give a counterexample to show that no such result is possible for quasiconformal images of (inner) $\alpha$-mcigar domains if $\alpha>0$. This counterexample $G$ is a broad inner $\alpha$-mcigar domain (and hence an inner $\alpha$-wslice ${ }^{+}$domain by [BS2, Lemma 3.4]), but it has a quasiconformal image which is not an $\alpha$-wslice domain. The construction of $G$ is rather intricate, but we suspect that this level of difficulty is inevitable. First, let us recall the definitions of conformal modulus and broadness.

The conformal modulus, $\bmod (E, F ; G)$, of the pair of disjoint compact subsets $E, F$ of a domain $G \subset \mathbb{R}^{n}$ is the infimum of $\int_{G} \rho^{n}$, as $\rho: G \rightarrow[0, \infty]$ ranges over the class of Borel functions for which every line integral over a path $\gamma:[0,1] \rightarrow G$ joining $E$ and $F$ is at least 1 . We refer the reader to $\mathrm{V} 2$ for the fundamentals of conformal modulus and quasiconformal mappings. 
A domain $G \subset \mathbb{R}^{n}$ is $\phi$-broad if

$$
\phi(t) \equiv \inf \left\{\bmod (E, F ; G): \Delta_{G}(E, F) \leq t\right\}>0, \quad t>0,
$$

where $E, F$ designate non-degenerate disjoint continua in $G$ and

$$
\Delta_{G}(E, F) \equiv \frac{d_{G}(E, F)}{\operatorname{dia}_{G}(E) \wedge \operatorname{dia}_{G}(F)}
$$

denotes the relative inner distance between $E$ and $F$. This term was introduced by Väisälä [V4, 2.15]. Our definition looks a little weaker than Väisälä's, but is equivalent to it by [HK, Theorem 3.6]. It is a special case of what is termed a Loewner space, but we prefer the term "broad" as it implicitly indicates that we are using the inner Euclidean metric.

A good way of starting this process of creating a counterexample is to fix $\alpha_{0} \in$ $(0, \alpha)$, and to recall Lappalainen's example $[\mathrm{L}, 6.7]$ of a planar set $D$ which is in the class $s$-mcigar for all $s>\alpha_{0}>0$ but not for any $s \leq \alpha_{0}$. These examples $D$ have two major defects that must be rectified before they can provide a counterexample: they are slice domains (since they are simply connected), and they are not broad. By Theorem 4.1 in BK2, one way of killing the slice and $s$-wslice ${ }^{+}$properties for $s \leq \alpha_{0}$ while preserving it for $s=\alpha$ is to replace $D$ by $D \times(0,1)$. This process does not suit us here because $D \times(0,1)$ is not broad. Instead, we "stack" copies of $D$ on top of each other. The word "stack" is meant to be more suggestive than accurate: since we want a broad domain, our new domain must have a vertical cross-section whose length is much less variable than that of $D$. To achieve this, we vary the numbers of stacked copies of $D$ as we move from left to right - a "copy" of $D$ bifurcates into two copies roughly where the cross-sectional height halves. Also, to ensure that the domain is broad, we need very gappy barriers between stacked layers; in fact we shall choose a sequence of isolated points as "barriers".

Our approach is therefore as follows. Fixing $0<\alpha_{0}<\alpha<1$, we first construct a variant $D$ of Lappalainen's example of a planar set which lies in the class $s$ mcigar for all $s>\alpha_{0}$ but not for any $s \leq \alpha_{0}$. Next we define a "quantized", more easily stackable, version of $D$ whose cross-sectional heights are similar to those of $D$, but are always powers of 2 . Finally we define our stacked set $G$ and find a quasiconformal mapping that maps $G$ to another domain $\Omega$ that does not possess an $\alpha$-wslice ${ }^{+}$property.

Before beginning, let us introduce some notation and terminology that we shall

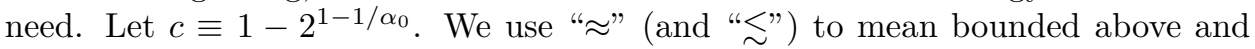
below (or just above, respectively) by constants dependent only on $\alpha$ and $\alpha_{0}$. For any function $f:(0,1) \rightarrow[0, \infty)$, we denote by $D_{f}$ the planar set $\left\{(x, y) \in \mathbb{R}^{2}\right.$ : $0<x<1,|y|<f(x)\}$. Every such $f$ that we use will be a symmetric function, meaning that it will satisfy the symmetry relation $f(x)=f(1-x), 0<x<1$, so it suffices to define $f(x)$ (or $\left.D_{f}\right)$ for $0<x \leq 1 / 2$. Let $d_{1}$ denote the metric associated with the $l^{1}$-norm $\|(x, y)\|_{1}=|x|+|y|$. Given a line segment $J=[z-R, z+R]$ on the $x$-axis, $z=(x, 0), R=(r, 0)$, we denote by $B_{1}(J)$ the metric ball $B_{d_{1}}(z, r)$; note that $B_{1}(J)$ is a "diamond" whose intersection with the $x$-axis is $J$.

By the regular $c$-Cantor set, $A_{c}$, we mean the set that remains when one starts with the single zeroth stage interval $(0,1)$, and iteratively removes a subinterval of length $c|I|$ from the center of each subinterval that remains at the $n$th stage, $n \geq 0$. We denote by $I_{n, j}, 1 \leq j \leq 2^{n}$, the $2^{n}$ open intervals removed at the $n$th stage, viewed as intervals on the $x$-axis of the plane. Let $z_{n, j}=\left(x_{n, j}, 0\right)$ be the center, 
and $r_{n}=c 2^{-1-n / \alpha}$ the half-length, of $I_{n, j}$; we assume the labeling is done so that $\left(x_{n, j}\right)_{j=1}^{2^{n}}$ is an increasing sequence. We are particularly interested in $z_{n}^{-}=\left(x_{n}^{-}, 0\right)$ and $z_{n}^{+}=\left(x_{n}^{+}, 0\right)$, the left and right-hand endpoints of $I_{n, 1}$.

We now construct $D$, which decomposes as the union of two open subsets $\widehat{D}$ and $D^{*}$, where

$$
\widehat{D}=\bigcup_{n=0}^{\infty} \bigcup_{j=1}^{2^{n}} B_{1}\left(I_{n, j}\right)
$$

is an affine copy of Lappalainen's original $D^{\prime}$, but $D^{*}$ (defined below) is rather different from his $D^{*}$. Note that $\widehat{D}=D_{\hat{f}}$ for some function $\hat{f}$, and that $\hat{f}^{-1}(0) \equiv A_{c}$ has $H^{1}$ measure zero; the set $\widehat{D}$ is illustrated in Figure 5.1 (to a resolution of $n=3$ ). As in [L, 6.7], a straightforward calculation shows that

$$
\int_{I_{n, j}} \delta_{\widehat{D}}^{\beta-1}(z) d z=K^{\prime} r_{n}^{\beta}, \quad \beta>0
$$

where $K^{\prime}=K^{\prime}\left(\beta, \alpha_{0}\right)<\infty$. In particular, $d_{\alpha, \bar{D}}\left(z_{n}^{-}, z_{n}^{+}\right)=2 K r_{n}^{\alpha}$, where $K=$ $K^{\prime}\left(\alpha, \alpha_{0}\right) / 2$.

We next define $D^{*}$. Our $D^{*}$ is different than that of Lappalainen, but both sets have a similar role: they connect the components of $\widehat{D}$ to make $D$ a domain, while being so highly cusped that certain desirable metric properties of $\widehat{D}$ are inherited by $D$. Note that $D$ is a domain as long as $D^{*}=D_{f^{*}}$, for some function $f^{*}:(0,1) \rightarrow(0, \infty)$ whose only discontinuities are jump discontinuities at points $x$ where both one-sided limits of $f^{*}$ are less than $\hat{f}(x)$. We construct such a symmetric function $f^{*}$, defined on $(0,1 / 2]$ as follows. First we choose $f^{*}(x)=c_{1}$ for all $x \in\left(x_{1,1}, 1 / 2\right]$, where $c_{1}$ is a constant. Note that $d_{\alpha, D}\left(z_{2}^{-}, z_{2}^{+}\right)$is independent of $\left.f^{*}\right|_{\left(0, x_{1,1}\right)}$ and increases ever closer to $2 K r_{2}^{\alpha}$, as $c_{1} \rightarrow 0^{+}$. Thus we can choose $c_{1} \in\left(0, \hat{f}\left(x_{1,1}\right)\right)$ so small that $d_{\alpha, D}\left(z_{2}^{-}, z_{2}^{+}\right) \geq K r_{2}^{\alpha}$. Inductively, for each $n \geq 2$, we choose $f^{*}(x)=c_{n}$ for all $x \in\left(x_{n, 1}, x_{n-1,1}\right]$, where $c_{n} \in\left(0, \hat{f}\left(x_{n, 1}\right)\right)$ is so small that $d_{\alpha, D}\left(z_{n+1}^{-}, z_{n+1}^{+}\right) \geq K r_{n+1}^{\alpha}$, and hence

$$
K r_{n}^{\alpha} \leq \int_{I_{n, j}} \delta_{D}^{\alpha-1}(z) d z \leq 2 K r_{n}^{\alpha}, \quad 1 \leq j \leq 2^{n}
$$

As in [L, 6.7], it easily follows that $D$ is an $\alpha$-mcigar domain.

Note that $D=D_{f}$, where $f=\hat{f} \vee f^{*}$. By construction $f(x) \leq C[x \wedge(1-x)]$, with $C=2 f(1 / 2) \leq 1$; equality in the first inequality occurs at all points of the form $z_{n, j}, j=1$ or $2^{n}$. Moreover, $|f(y)-f(x)| \leq|y-x|$, with equality for $x, y$ near $1 / 2$, but less than $1 / 2$; more briefly, we write $\|f\|_{\operatorname{Lip}_{1}((0,1))}=1$.

We now "quantize" $D$ to get a new domain $D_{g}, g$ being a function whose values lie in the set $S=\left\{2^{-n-1}: n \in \mathbb{Z}\right\}$. For an appropriate strictly decreasing sequence of positive numbers $\left\{x_{j}\right\}_{j=0}^{\infty}, g$ will be a lower semicontinuous symmetric function which takes on a constant value $t_{j} \in S$ on each interval $\left(x_{j+1}, x_{j}\right)$; lower semicontinuity is to ensure that $D_{g}$ is open. First $x_{0}=1 / 2$ and $t_{0}$ is the smallest element of $S$ which exceeds $f(1 / 2) / 4$. For $k>0, t_{k}$ will always be $f\left(x_{k}\right)$, so we shall define $x_{k}$ only. First, $x_{1}$ is the point $x$ closest to $x_{0}$ where $f(x)=t_{0} / 2$. Inductively, assume that we have defined $x_{j}$ for all $j \leq k$. As we travel from $x_{k}$ to 0 we reach points $x$ where $f(x) \in S$. Then $x_{k+1}$ will be the first such point that satisfies the following 

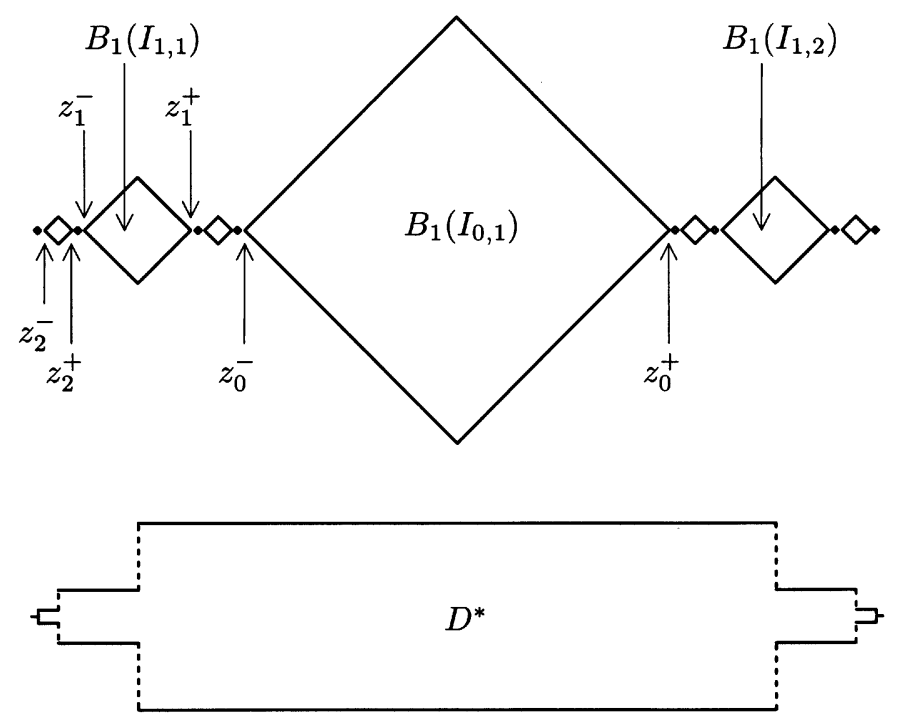

Figure 5.1. The set $\widehat{D}$ (top) and domain $D^{*}$ (both for $\alpha_{0}=1 / 2$ )

stopping time condition:

(1) either $f(x)=t_{k} / 2$, or

(2) $f(x)=2 t_{k}$ and $f(y) \geq f(x)$ for all $y \in[x-f(x) / 2, x]$.

Since we already proved that $f(x) \leq x$, it follows that $x_{k+1}$ will always be defined. As we let $x$ move from $x_{k}$ towards 0 , we may reach a point $x^{\prime}$ such that $f\left(x^{\prime}\right)=2 t_{k}$, but the other part of (2) fails to hold. In this case, the Lipschitz continuity of $f$ implies that $f(y)<5 t_{k} / 2$ for all $y \in\left[x^{\prime}-f\left(x^{\prime}\right) / 2, x^{\prime}\right]$. Thus on the interval $\left[x_{k+1}, x_{k}\right], t_{k} / 2 \leq f \leq 4 t_{k}$ (the upper bound can only be approached near $x_{0}$ ), and on the corresponding open interval, $g \equiv t_{k}$. Combining these facts with the continuity of $f$ and lower semicontinuity of $g$, we obtain the uniform estimate: $1 / 2 \leq f / g \leq 4$, and so

$$
d_{\alpha, D_{g}}\left(z_{n}^{-}, z_{n}^{+}\right) \approx r_{n}^{\alpha}, \quad n \geq 2 .
$$

The Lipschitz bound on $f$ also implies that $x_{j-1}-x_{j} \geq f\left(x_{j-1}\right) / 2$. It follows that for $j \in \mathbb{N}, x_{j-1}-x_{j} \approx t_{j}$ since if $x_{j-1}-x_{j}$ were much larger than $f\left(x_{j-1}\right)$, then $D_{f}$ would have a long thin corridor, a feature that $\alpha$-mcigar domains such as $D_{f}$ cannot have.

Before defining our counterexample, we need to define some auxiliary sets $U, V, W$ (illustrated in Figures 5.3 and 5.4), all symmetric about the line $x=1 / 2$. First, $U=D_{u}$, where $u(x)=x \log _{2}(1 / x)$, when $x \leq 1 / 2$. The left-half of $V$ is given by

$$
V \cap((0,1 / 2] \times \mathbb{R})=U \backslash \bigcup_{m \in \mathbb{Z} \backslash\{0\}} \bigcup_{j=0}^{\infty}\left[\left(x_{j+1}, m t_{j}\right),\left(x_{j}, m t_{j}\right)\right] .
$$

$W$ is the disconnected open subset of $V$ obtained by additionally removing the vertical lines $\left\{x_{j}\right\} \times \mathbb{R},\left\{1-x_{j}\right\} \times \mathbb{R}, j \geq 0$. We define $W$-cells to be the components of $W$. Note that the width, $x_{j}-x_{j+1}$, and height, $t_{j}$ or $2 t_{j}$, of any rectangular 


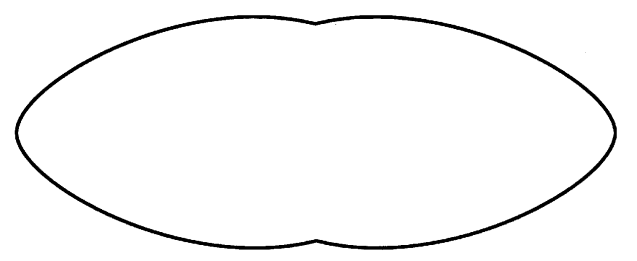

FiguRE 5.3. The domain $U$

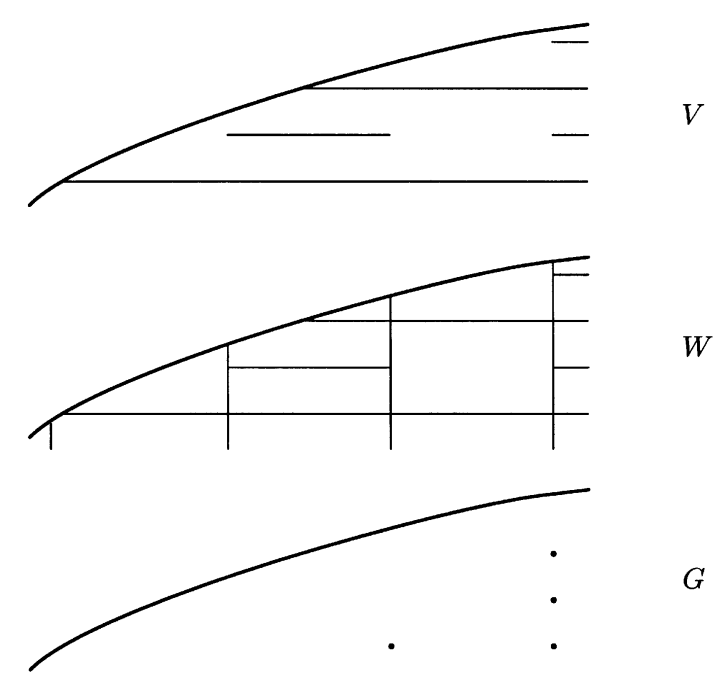

Figure 5.4. Close-ups of $V, W, G$ near $\partial U$

$W$-cell are approximately equal, and so rectangular $W$-cells are $C$-uniform domains for some $C=C\left(\alpha, \alpha_{0}\right)$.

We now define $G$ to be the set obtained by removing from $U$ those corners of $W$-cells that are corners only of rectangular $W$-cells (i.e. they are not too close to the boundary of $U$; see Figure 5.4), and we define a $(G$-)cell to be the $G$-closure of any $W$-cell. We say that a point $z$ in a cell $A$ of $G$ is horizontally (or vertically) central if it lies on the vertical (or horizontal) line that bisects $A$. It is not hard to see that the domain $U$ is (inner) uniform, and hence broad by a special case of Example 6.5(b) in [BHK]. Since $U \backslash G$ is countable, it is a null set for extremal distance $[\mathrm{V} 1$ and $\operatorname{so} \bmod (E, F ; G)=\bmod (E, F, U)$ for all disjoint compact subsets of $G$. Thus $G$ is also broad.

To show that $G$ is an $\alpha$-wslice ${ }^{+}$domain, it suffices by [BS2] Lemma 3.4] to show that $G$ is an $\alpha$-mcigar domain. We first define an equivalence relation $\sim$ on $V$ by $z \sim w$ if there is a path $\gamma$ from $z$ to $w$ whose first coordinate is a monotone function; roughly speaking $z \sim w$ if $z, w$ lie in the same stacked copy of $D_{g}$. It is easy to find an $\alpha$-mcigar path for a pair of points in the same cell, or in a pair of cells that share a boundary point, so we assume the points are further apart than this. One can efficiently move from any point in a cell to the center of that cell, so it suffices 
to find an $\alpha$-mcigar path for a pair $z=(x, y)$ and $w=(u, v)$ of cell centers (which automatically lie in $V$ ). We shall find $\alpha$-mcigar paths separately for "horizontal" pairs (meaning that $z \sim w$ ) and "vertical" pairs $z, w$ (meaning that $x=u$ ). By the triangle inequality, it then follows that $\alpha$-mcigar paths exist for any pairs of cell centers.

If $z \sim w$, it is not hard to see that we can connect $z$ and $w$ in $V$ by a piecewise straight path $\gamma$ whose slope never exceeds 1 and for which the distance between $\gamma(t)$ and the nearest boundary points of $V$ directly above and below $\gamma(t)$ are within a factor 3 of each other; we omit the details. By reference to the $\alpha$-mcigar domain $D_{g}$, we see that $\gamma$ is an $\alpha$-mcigar path for $z, w$ in $V$, and consequently also in $G$.

Suppose, on the contrary, that $z, w$ is a vertical pair. Let $z^{\prime}=\left(x^{\prime}, y^{\prime}\right)$ be a vertically central point such that $z^{\prime} \sim z, x^{\prime} \in[x, 1 / 2]$, and $\left|x-x^{\prime}\right|=|z-w| \wedge|x-1 / 2|$. Similarly, let $w^{\prime}$ be a vertically central point with first coordinate $x^{\prime}$ for which $w^{\prime} \sim w$. Let $\gamma_{z}$ be an $\alpha$-mcigar path from $z$ to $z^{\prime}$, and $\gamma_{w}$ be an $\alpha$-mcigar path from $w$ to $w^{\prime}$, with the parametrizations chosen so that the first coordinate of $\gamma_{z}(t)$ and $\gamma_{w}(t)$ are always equal.

Simple estimation shows that the $\alpha$-mcigar condition rules out any corridor whose cross-sectional width is much smaller than its length over a piece whose length is some reasonable fraction of the total length of the corridor; for a more precise statement of this phenomenon, take $\beta=\alpha$ in [BK2, Lemma 2.2]. It follows from that result that there exist points $z^{\prime \prime} \equiv \gamma_{z}(t), w^{\prime \prime} \equiv \gamma_{w}(t)$ for some $t$, such that $z^{\prime \prime}$ is horizontally central, such that $\delta_{V}\left(z^{\prime \prime}\right)$ (and hence the height of the cell in which $z^{\prime \prime}$ lies) exceeds $|z-w| / C$ for some $C=C\left(\alpha, \alpha_{0}\right)>0$, and such that $w^{\prime \prime}$ has analogous properties. We now define a path $\gamma$ from $z$ to $w$ by gluing together three subpaths: first $\left.\gamma_{z}\right|_{[0, t]}$, then the vertical line segment from $z^{\prime \prime}$ to $w^{\prime \prime}$, and finally the path $\left.\gamma_{w}\right|_{[0, t]}$ run backwards. We leave it to the reader to verify that $\gamma$ is an $\alpha$-mcigar path relative to $G$.

It remains to specify a quasiconformal mapping $F: U \rightarrow \mathbb{R}^{2}$ such that $\Omega \equiv$ $F(G)$ does not satisfy an $\alpha$-wslice property. Letting $H$ denote the left-half of $G$, $H=\{(x, y) \in G \mid x \leq 1 / 2\}$, we claim that the continuous function $F=\left(F_{1}, F_{2}\right)$ : $U \rightarrow \mathbb{R}^{2}$ satisfying the symmetry property

$$
F(1-x, y)=\left(2-F_{1}(x, y), F_{2}(x, y)\right), \quad(x, y) \in U
$$

and defined on $H$ by the equation

$$
F(x, y)=\left(\frac{1}{\log _{2}(1 / x)}, \frac{y}{x \log _{2}^{2}(1 / x)}\right), \quad(x, y) \in H
$$

is such a function. To justify our claim, we define $G_{n}=\left\{(x, y) \in G \mid 2^{-n-1}<x \leq 2^{-n}\right\}$, $n \in \mathbb{N}$, so that $H=\bigcup_{n=1}^{\infty} G_{n}$. Except for a finite number of removed points, $F\left(G_{n}\right)$ is a quadrilateral with corners $(1 /(n+1), \pm 1 /(n+1))$ and $(1 / n, \pm 1 / n)$, and $\left.F\right|_{G_{n}}=b_{n} \circ d_{n}$ where $d_{n}$ is a dilation by a factor $2^{n} / n^{2}$, and $b_{n}$ is a bilipschitz map which distorts distances by at most a factor 8 . Since $F$ is smooth, this local factorization implies that it is $K$-quasiconformal for some universal $K$. It also follows that

$$
\operatorname{len}_{\alpha, \Omega}(F \circ \gamma) \approx 2^{n \alpha} n^{-2 \alpha} \cdot \operatorname{len}_{\alpha, G}(\gamma)
$$


whenever $\gamma$ is a path that lies in $G_{n}$. By routine estimation and (5.2), we see that

$$
d_{\alpha, G}\left(z_{n}^{-}, z_{n}^{+}\right) \approx d_{\alpha, D_{g}}\left(z_{n}^{-}, z_{n}^{+}\right) \approx r_{n}^{\alpha}, \quad n \geq 2 .
$$

Since $x_{n}^{-} \approx x_{n}^{+} \approx r_{n} \approx 2^{-n / \alpha},(5.5)$ implies that

$$
d_{\alpha, \Omega}\left(F\left(z_{n}^{-}\right), F\left(z_{n}^{-}\right)\right) \approx \frac{2^{n}}{(n / \alpha)^{2 \alpha}} \cdot 2^{-n} \approx n^{-2 \alpha} .
$$

Let $w_{k} \equiv\left(2^{-k}, 0\right)$. If $k \in \mathbb{N}$ is large, then any path $\lambda$ in $\Omega$ from $w_{k}$ to $w_{k+1}$ has to pass through approximately $2^{k}$ strips $\left(F_{1}\left(z_{n}^{-}\right), F_{1}\left(z_{n}^{+}\right)\right) \times \mathbb{R}$, all with $n \approx 2^{k}$, and so the $d_{\alpha, \Omega}$-length of the part of $\lambda$ in any one of these strips must be comparable to, or exceed, $n^{-2 \alpha}$. Thus $d_{\alpha, \Omega}\left(w_{k}, w_{k+1}\right) \geq 2^{k(1-2 \alpha)}$. As $k$ tends to infinity, this distance becomes much larger than $2^{-k \alpha}$, thus proving that $\Omega$ is not an $\alpha$-mcigar domain.

Now $F(U)$ is a triangle, so it certainly supports a $p$-Hölder imbedding for $p=$ $(n-\alpha) /(1-\alpha)$. Since the countable set of points deleted from $F(U)$ to form $\Omega$ is a removable set for $L^{1, p}$ (this follows from the ACL-characterization of Sobolev spaces [Mz, Section 1.1.3]), we conclude that $\Omega$ also supports a $p$-Hölder imbedding. This imbedding plus an $\alpha$-wslice condition would imply that $\Omega$ is an $\alpha$-mcigar domain according to [BS1, Theorem 0.1]. Since it is not an $\alpha$-mcigar domain, it is therefore not an $\alpha$-wslice domain.

\section{REFERENCES}

[BB] Z. Balogh and S.M. Buckley, Geometric characterizations of Gromov hyperbolicity, to appear in Invent. Math.

[BHK] M. Bonk, J. Heinonen, and P. Koskela, Uniformizing Gromov hyperbolic spaces, Astérisque 270 (2001), viii+99 pp. MR 2003b:30024

[B] S.M. Buckley, Quasiconformal images of Holder domains, preprint.

[BK1] S.M. Buckley and P. Koskela, Sobolev-Poincaré implies John, Math. Research Letters 2 (1995), 577-593. MR 96i:46035

[BK2] S.M. Buckley and P. Koskela, Criteria for Imbeddings of Sobolev-Poincaré type, Internat. Math. Res. Notices (1996), 881-901. MR 98g:46041

[BO] S.M. Buckley and J. O'Shea, Weighted Trudinger-type inequalities, Indiana Univ. Math. J. 48 (1999), 85-114. MR 2001a:46031

[BS1] S.M. Buckley and A. Stanoyevitch, Weak slice conditions and Hölder imbeddings, J. London Math. Soc. 66 (2001), 690-706. MR 2002h:46051

[BS2] S.M. Buckley and A. Stanoyevitch, Weak slice conditions, product domains, and quasiconformal mappings, Rev. Math. Iberoam. 17 (2001), 1-37. MR 2003b:30025

[GM1] F.W. Gehring and O. Martio, Lipschitz classes and quasiconformal mappings, Ann. Acad. Sci. Fenn. Ser. A I Math. 10 (1985), 203-219. MR 87b:30029

[GM2] F.W. Gehring and O. Martio, Quasiextremal distance domains and extension of quasiconformal mappings, J. Analyse Math. 45 (1985), 181-206. MR 87j:30043

[GO] F.W. Gehring and B. Osgood, Uniform domains and the quasihyperbolic metric, J. Analyse Math. 36 (1979), 50-74. MR 81k:30023

[HK] J. Heinonen and P. Koskela, Quasiconformal maps in metric spaces with controlled geometry, Acta Math. 181 (1998), 1-61. MR 99j:30025

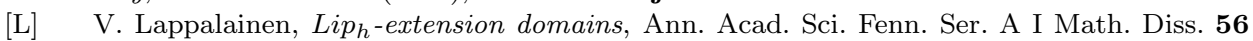
(1985), 1-52. MR 87h:26021

[Mz] V.L. Maz'ya, Sobolev Spaces, Springer-Verlag, Berlin, 1985. MR 87g:46056

[V1] J. Väisälä, On the null-sets for extremal distances, Ann. Acad. Sci. Fenn. Ser. A I 322 (1962), 12pp. MR 26:5148

[V2] J. Väisälä, Lectures on n-dimensional quasiconformal mappings, Lecture Notes in Mathematics 229, Springer-Verlag, Berlin, 1970. MR 56:12260

[V3] J. Väisälä, Uniform domains, Tohoku Math. J. 40 (1988), 101-118. MR 89d:30027 
[V4] J. Väisälä, Quasiconformal mappings of cylindrical domains, Acta Math. 162 (1989), 201225. MR 90f:30034

[V5] J. Väisälä, Relatively and inner uniform domains, Conf. Geom. Dyn. 2 (1998), 56-88. MR 99e:30014

Department of Mathematics, National University of Ireland, Maynooth, Co. KilDARE, IRELAND

E-mail address: sbuckley@maths.may.ie

Division of Mathematical Sciences, University of Guam, Mangilao, Guam 96923, USA

E-mail address: alex@math.hawaii.edu 